\title{
Philosophiques
}

\section{Jean-Jacques Rousseau et Friedrich Schiller : le théâtre sous le feu des lumières}

\section{Martin Matte}

Volume 17, numéro 2, automne 1990

URI : https://id.erudit.org/iderudit/027122ar

DOI : https://doi.org/10.7202/027122ar

Aller au sommaire du numéro

Éditeur(s)

Société de philosophie du Québec

ISSN

0316-2923 (imprimé)

1492-1391 (numérique)

Découvrir la revue

Citer cet article

Matte, M. (1990). Jean-Jacques Rousseau et Friedrich Schiller : le théâtre sous le feu des lumières. Philosophiques, 17(2), 101-145.

https://doi.org/10.7202/027122ar
Résumé de l'article

Une forme d'art comprend-elle le critère de son acceptation ou de son rejet par la société dans laquelle elle prend forme? Le public auquel une oeuvre d'art s'adresse possède-t-il la compétence de faire l'exploration qu'elle lui propose? La Lettre à d'Alembert sur les spectacles de Rousseau et la Conférence de Schiller : "Was kann eine gute stehende Schaubùhne eigentlich wirken? » donnent chacune réponse à ces deux questions par la caractérisation d'une manière de sentir et d'agir propre aux protagonistes du domaine esthétique. Je propose d'examiner si les principes sur lesquels l'un appuie sa condamnation de la représentation théâtrale, l'autre son apologie de l'efficace de la scène, ont leur source et leur valeur dans l'expérience du plaisir du théâtre. 


\title{
JEAN-JACQUES ROUSSEAU ET FRIEDRICH SCHILLER : LE THÉÂTRE SOUS LE FEU DES LUMIÈRES
}

\author{
par Martin Matte
}

RÉSUMÉ. Une forme d'art comprend-elle le critère de son acceptation ou de son rejet par la société dans laquelle elle prend forme ? Le public auquel une cuvre d'art s'adresse possède-t-il la compétence de faire l'exploration qu'elle lui propose? La Lettre à d'Alembert sur les spectacles de Rousseau et la Conférence de Schiller : «Was kann eine gute stebende Scbaubübne eigentlich wirken? „ donnent chacune réponse à ces deux questions par la caractérisation d'une manière de sentir et d'agir propre aux protagonistes du domaine esthétique. Je propose d'examiner si les principes sur lesquels l'un appuie sa condamnation de la représentation théâtrale, l'autre son apologie de l'efficace de la scène, ont leur source et leur valeur dans l'expérience du plaisir du théâtre.

ABSTRACT. Does a kind of art hold in itself the criterion of its acceptance or of its reject by the society in which it takes shape? Is the public to whom a work of art is aimed enabled to make the exploration it suggests to him ? The Lettre à d'Alembert sur les spectacles by Rousseau and the lecture of Schiller : "Was kann eine gute stebende Schaubübne eigentlich wirken? " give each one answer to both questions by the caracterisation of a way of feeling and working proper to aesthetic protagonists. I suggest to examine if the principles on which lay the reproof of the theatrical representation by Rousseau and the justification of the stage's efficacious by Schiller stem from and are worth according to the experience of theatrical enjoyment. 
"La nature humaine, au fond, n'était pas meilleure; les hommes trouvaient leur sécurité dans la facilité de se pénétrer réciproquement ».

Rousseau, Premier Discours ${ }^{1}$.

La discussion autour de la fonction du théâtre a été, au XVIII ${ }^{e}$ siècle, l'occasion de mettre en présence les trois acteurs maintenant manifestes de la pratique esthétique : les auteurs, les ouvres et le public. Rousseau, dans sa Lettre à d'Alembert sur les spectacles, et Schiller, dans sa conférence "Que pourrait être l'effet véritable d'une bonne scène permanente ${ }^{2}$, me paraissent y avoir contribué d'une façon intéressante par la caractérisation d'une manière de sentir et d'agir propre aux protagonistes du domaine esthétique. Leurs réponses respectives au problème de l'acquisition par les membres d'une société de la compétence de faire l'exploration que les ouvres d'art leur proposent ou d'en jouir sont cependant diamétralement opposées. En vue d'examiner la pertinence de chacune, je me propose de discerner si les principes sur lesquels l'un et l'autre s'appuient ont leur source et leur valeur précisément dans ce qui a lieu dans l'expérience du théâtre. Outre l'histoire de la fonction de celui-ci, j'aurai recours au concept de jouissance esthétique de H.R. Jauss, pionnier de l'esthétique de la réception ${ }^{3}$, pour mettre en relief comment l'expérience esthétique n'est pas indifférente au bonheur d'une société.

\section{Rousseau, Schiller et la question du théâtre}

La réplique de Rousseau à d'Alembert au sujet du "projet d'établir un théâtre de comédie à Genève» ( $L$. d'A., p. 156), proposé au public dans l'article «Genève » du tome

1. ROUSSEAU, Jean-Jacques, Discours sur les sciences et les arts (1751), Lettre à d'Alembert sur les spectacles (1758), édition établie et présenté par Jean Varloot, Paris, Gallimard, 1987, p. 47. Ci-après $L d ' A$, La Lettre va des pages $137 \gtrsim 312$.

2. SCHILLER, Friedrich, Säntlicbe Werke, fünfter Band, *Erzäblungen/Theoretische Scbriften*, München, Karl Hanser Verlag, 1962: „Die Schaubübne als moralische Anstalt betrachtet $*(1802)=\star$ Was kann eine gute stebende Schaubübne eigentlicb wirken ?* (1785), p. 818-831. Ci-après $S$. Mes remerciements chaleureux vont à Christa Schebesch de Berlin pour sa vigilante révision de ma traduction. C'est sur son aide amicale que j'appuie librement mon interprétation.

3. On trouvera, en français, ce concept dans : Hans Robert JAUSS, * La jouissance esthétique , in Poetique, 39, septembre 1979, p. 261-274, et * Petite apologie de la jouissance esthétique $*$, in Pour une estbétique de la réception, rr. Fr. Claude Maillard, Paris, Gallimard, 1978, p. 123-158. L'idée d'une esthétique de la réception est expliquée dans * Esthétique de la réception et communication litteraire , in Critique, 413, octobre 1981, p. 1116-1130. 
septième de l'Encyclopédie, plaide en faveur des spectacles simples et naturels sur la base d'un critère quasi juridique. Pour Rousseau, la "perfection" d'un divertissement public doit s'établir * le mieux possible selon la nature de l'homme et la constitution de la société * ( $L$. d'A., p. 279). Dans la dernière des trois parties de sa Lettre, il analyse la situation économico-sociale de Genève, en décrit les usages et conclut que cet établissement-là ruinerait l'équilibre social genevois. Voilà pourquoi il s'attache à démontrer que les spectacles n'ont rien d'indifférent, tant au point de vue de l'art, de la morale que de la société, ce cas d'application devant servir de règle à l'égard de toute autre société. Il n'appartient pas au théâtre de révéler les sentiments que les membres d'une société se manifestent ni les actions qu'ils entreprennent pour assurer l'autonomie de leur existence. Dans les termes de Rousseau, ce n'est pas à l'art qu'il revient d'agir sur les mœurs en changeant l'opinion publique. Au contraire, Schiller se porte à la défense des vérités propres à l'imaginaire théâtral. La scène constitue pour lui un accès privilégié aux jugements de l'histoire et il croit que les leçons de "sagesse pratique" $(S$, p. 824) que donne «la scène la meilleure" rendent son public apte à établir les médiations recherchées par tous entre la pulsion naturelle qui tend au plaisir et l'inconditionnelle raison qui nous incite à vouloir être libre. L'institution théâtrale peut aider à soustraire l'humanité à la contraignante nécessité du besoin. *Ainsi, écrit-il, celui qui peut prouver sans nulle contradiction que le théâtre contribue à l'éducation des hommes et du peuple a décidé du rang de celui-ci parmi les premières institutions de l'État" ( $S$., p. 819). Cet idéal de formation est nécessairement relié à la plus haute et à la dernière exigence que le philosophe et le législateur d'une institution publique peuvent faire, celle «de promouvoir le bonheur général" (id.). Le problème de la fonction sociale de l'art est ainsi ouvert à la fois par des prescriptions sur la vie des sociétés et par des réflexions sur l'action du théâtre.

Au moment d'établir "l'état de la question", Rousseau écrit : "Tout est problème encore sur les vrais effets du théâtre" (L. d'A., p. 157). Son débat avec ses contemporains porte, en effet, sur le dilemme classique de déterminer si l'art a pour fonction d'instruire ou de divertir. Comment instruire sans induire en erreur et comment divertir sans tomber dans l'abus? Si cela est inévitable, il ne s'agit pas pour y remédier, selon Rousseau, de demander «si les spectacles sont bons ou mauvais en eux-mêmes" (L. d'A., p. 159). S'ils étaient tous mauvais, il suffirait d'en "ôter la cause", au 
premier chef les comédiens. À cette question trop vague, il préfere opposer le principe que * les spectacles sont faits pour le peuple» (id.). Ce n'est que par leurs effets sur les hommes de tel temps ou de tel pays qu'on peut déterminer leurs qualités artistiques et chercher ce qui est moralement bon pour ceux-ci. Deux choses me semblent motiver ce relativisme pratique. La première veut découvrir les origines des spectacles dans leur destination, la seconde faire de cette destination le critère pour décider de la nécessité ou non d'interdire le théâtre, son institution. Rousseau a raison de dénoncer comme purement formelle la recherche de la fonction de l'art dominée par l'idée de la perfection du spectacle théâtral, mais sa dénonciation résulte par ailleurs en une subordination du jeu théâtral à sa réussite extra-esthétique. En effet, le même critère, celui de la transparence et de l'immédiateté, doit être applicable en politique, en morale et en esthétique. Rousseau ne connaît de société heureuse que celle qui se constitue d'après la nature des hommes et de leurs occupations. "L'état d'homme, écrit-il, a ses plaisirs, qui dérivent de sa nature, et naissent de ses travaux; et ces plaisirs, d'autant plus doux que celui qui les goûte a l'âme plus saine, rendent quiconque en sait jouir peu sensible à tous les autres" (L. d'A., p. 158). L'unique prescription, qui sert de critère de détermination de la nature de ces plaisirs, est de laisser « un peuple se délasser de ses travaux quand et comme il lui plaît» (L. d'A., p. 212). C'est pourquoi sa Lettre se termine avec la proposition du modèle des fêtes républicaines. Le divertissement théâtral paraît n'avoir pas d'autre légitimité que celle qu'une organisation librement consentie de la vie civile lui accorde.

C'est à bon droit que Schiller critique toute doctrine qui, dénonçant les effets du théâtre sur la société comme illusoires, dénie à celui-ci tout mérite artistique. Des affirmations qu'on a faites jusqu'à lui concernant le théâtre, y compris celles de Rousseau, bien que toutes soient «incontestablement vraies", aucune, écrit-il, "ne touche la scène" $(S .$, p. 820-821). Cette ambivalence de Schiller se reflète dans la double fonction qu'il attribue à "la scène la meilleure» (S., p. 828). Avant tout, il appelle à reconnaître qu'elle se situe hors de ce monde d'où peuvent être émises ces lois qui l'interdisent. Son action est indépendante de ces jugements qui se contredisent les uns les autres. «La juridiction de la scène, écrit-il, dans une formule fondatrice, commence là où les lois de ce monde prennent fin» (S., p. 823). En identifiant la scène à un "redoutable tribunal», il autonomise l'art du théâtre et confère l'impunité à ceux qui le 
pratiquent, auteurs, comédiens et spectateurs, au moment où tous sont réunis pour la présentation d'une œuvre scénique. Le problème de Schiller est de prouver la nécessité de cette autonomie. Pour sauvegarder l'indépendance de la scène, il faut attribuer une certaine permanence à ses effets ${ }^{4}$. La présence de la scène dans la cité ne peut être légitime que si ce qu'on y présente, s'y rendant publiquement accessible, acquiert une portée universelle. Schiller ne répond pas ainsi seulement au pragmatisme de Rousseau sur les divertissements théâtraux, il ouvre la question de la représentation sur la scène des voies par lesquelles une société se rend socialement responsable du bonheur de tous. L'efficacité qu'il octroie à la scène, il me semble cependant la gagner aux dépens du public du théâtre, sinon par la constitution de son propre public. Les spectateurs du théâtre moderne sont, en fait, réduits, devant la scène, à la passivité, alors qu'ils sont invités, au nom de la transcendance morale, à l'action dans la vie civile. C'est presque malgré eux que les spectateurs du siècle des "Lumières" se sont refusés à devenir un public de métier, "policé ", "attentif, calme et réfléchi " 5 . Ils ont semblé préférer se divertir au théâtre comme ailleurs, plutôt que d'éprouver la tension morale entre le monde enchanté de la scène et la forme de vie dominante ${ }^{6}$.

À première vue, Rousseau et Schiller ont à faire avec la double appartenance du sentiment à la réceptivité et à la spontanéité. Ils ne se posent pas encore le problème, que la troisième Critique de Kant héritera des difficultés de l'esthétique du XVIII' siècle, dans les termes d'un statut intermédiaire du jugement entre sensibilité et raison, désir et conscience, contingence et nécessité, nature et liberté.

4. KOSELLECK, Reinhart, (Le Regne de la critique, Paris, Minuit, 1979) explique la formule de Schiller comme le moyen d'accorder à rout le domaine social la liberté déjà conquise dans la * République des Letres * : la scène met en contact les lois existantes avec la nouvelle juridiction théAtrale wagissant au nom du sentiment humain $\approx$, p. $83 \mathrm{ssq}$.

5. KREBS, Roland, we théatre de l'Aufklärung. Un theAtre à la recherche de son public, , in Recbercbes nouvelles sur l'Aufklär'ung, Actes du 18 e Congrès de l'Association des Germanistes de l'Enseignement supérieur, Reims 27-28 avril 1985, publiés par Roland Krebs, Reims, Presses Universitaires de Reims, 1987 , p. 5-23.

6. GADAMER, H.-G., *Über die Festlichkeit des Theaters*, in Kleine Scbriften II, Interpretationen, Tübingen, J.C.B. Mohr (Paul Siebeck), 1967, p. 170-178. Traduction anglaise : * The festive character of theater *, in H-G. Gadamer, The Relevance of Beautiful and other essays, éd. par Bernard Bernasconi, tr. Nicolas Walker, Cambridge, Cambridge University Press, 1986, p. 57-65. Ce texte reprend celui de la conférence prononcée en 1954 pour la commémoration du $175^{\mathrm{e}}$ anniversaire de la fondation du theatre national Schiller a Mannheim. J'y puise mes considérations sur l'histoire de la fonction du theatre. 
Tablant sur l'affinité des goûts et des mœurs, Rousseau dénie *au théâtre le pouvoir de changer des sentiments [et] des mours qu'il ne peut que suivre et embellir * ( $L$. $d^{\prime} A$., p. 161). Au contraire, misant sur sa situation intermédiaire entre sensualité et intelligence, Schiller établit que, seul, «le sens esthétique ou encore le sentiment pour le beau * provoque «ces résultats» $(S .$, p. 821), qu'un monde transformé par la distinction des fonctions humaines et des sphères d'activité attend. L'«état d'homme et l'institution sociale entrent en lutte sur le terrain de l'esthétique. Chez Schiller, le "sens esthétique* a, entre le manque et l'excès, une fonction inverse à celle que remplit l'action pour l'instinct d'activité. Son grand art est d'harmoniser les "tendances extrêmes et contraires * et de ménager * un passage réciproque d'une disposition à l'autre * (id.). Mais, pour Rousseau, c'est "une vérité de pratique importante à tout un peuple" (L. d'A., p. 143), qui doit permettre de décider ce qui convient à un peuple dans ce qui détermine sa vie. Son opposition au projet voltairien, rationaliste, d'implanter un théâtre à Genève est de part en part animée du désir, non de plaire aux Genevois, mais de tenir compte de leur cas particulier et de leur rendre justice ${ }^{7}$.

La découverte de la puissance du «sentiment", selon l'interprétation d'ensemble de la pensée de Rousseau par Cassirer, sert de principe à deux orientations différentes ${ }^{8}$. La première commande une attitude d'abandon à la nature, qui doit permettre d'en comprendre sans intermédiaire toute la plénitude et d'en jouir; l'autre en commande une de vouloir spontané du droit, ce vouloir originant de la conscience de la liberté, elle-même indissociable de l'idée du droit. Rousseau exclut ainsi le sentiment tout court comme fondement de la morale au profit de la loi, parce que l'accès aux vérités du domaine pratique exige seulement l'autonomie de la conscience, comme le vicaire savoyard le professe9. Le sentiment esthétique représente-t-il une troisième orientation, commandant une attitude mi-active mipassive? Si elle doit exister, Rousseau ne croit pas qu'elle passe par le détour de la scène. Tout peuple doit baigner

7. Il érait évident, meme pour Rousseau, qu'établi dans les environs de Genève Voltaire était l'instigateur le plus prochain de la proposition de d'Alembert, qui l'y avait visité en 1755 lors de son séjour en Suisse. Cf. Margaret M. MOFFAT, Rousseau et la querelle du théatre au XVIIIe siècle, Paris, 1930, Genève, Slatkine Reprints, 1970, p. 62.

8. CASSIRER, Emst, Le Probleme Jean-Jacques Rousseau, tr. fr. Marc B. de Launay, coll. Textes du XXe siecle, Paris, Hachette, 1987, p. 94 ssq.

9. ROUSSEAU, Jean-Jacques, Emile, Livre quatrième, "Profession de foi du vicaire Savoyard „, Paris, Garnier-Flammarion, 1966, p. 345 ssq. Ici, p. 376-377-378. 
dans une véritable immédiateté, celle dans laquelle se révèle à chacun le sentiment de ce qu'il est et où chacun prend connaissance de la loi d'autonomie qui régit sa conduite ${ }^{10}$. Si l'art engendre un sentiment ou s'il est engendré dans un sentiment, celui-ci doit toujours demeurer subordonné à la moralité. Rousseau n'affirme rien d'autre, en écrivant: "C'est du beau moral qu'il est ici question. Quoiqu'en disent les philosophes, cet amour est inné dans l'homme, et sert de principe à la conscience* ( $L . d^{\prime} A$., p. 167, note). Cette subordination témoigne de la difficulté de vouloir appliquer au domaine esthétique le principe d'immédiateté qui prévaut dans le domaine éthique ${ }^{11}$. Le théâtre et l'assemblée générale commandent deux attitudes bien distinctes de représentation. L'un et l'autre ne se laissent pas confondre sans dissonance dans le registre affectif du monde de la vie. La communauté affective de ceux qui s'abandonnent à la fête, dont Rousseau prévoit l'organisation pour eux, est en concurrence, je crois, avec la fusion des volontés de ceux qui, formés sous le modèle du Contrat social, s'assemblent pour délibérer ${ }^{12}$.

À l'inverse, résolvant par la recomposition de la "poétique" de la scène le paradoxe d'une passion active, Schiller affirme la légitimité du sentiment esthétique qu'une institution publique a moralisé. La conclusion qui, selon Koselleck, se dégage de sa réponse à ce que l'on peut attendre du théâtre considéré comme institution morale est "que le théâtre doit ménager chez les hommes une place pour le sentiment d'être un homme» ${ }^{13}$. Par la mise en correspondance de la science et du désir, Schiller ouvre la voie d'exploration de l'imaginaire humain et de ses modes de production, persuadé que les «tableaux présentés sur la scène se confondent à la fin avec la morale de l'homme ordinaire et déterminent son sentiment en des cas particuliers » $(S$. , p. 823). Mais là où la transparence de la vision et de la sensation, selon l'interprétation de la solution de Rousseau par Jauss, n'est pas ce qui suffit à fonder l'«obli-

10. CASSIRER, op. cit., p. 102.

11. C'est a juste titre que Philip KNEE, dans son article *Agir sur les coeurs spectacle et duplicité chez Rousseau *, in Pbilosopbiques, XIV, 2, automne 1987 p. 299-329, mentionne comment Rousseau récupère politiquement le modèle de la fete, p. 314-315. Cet article analyse le prestige du paraître theatral J'y renvois le lecteur pour cet aspect de la question des spectacles qui ne me retiendra pas ici.

12. CF. STAROBINSKI, Jean, Jean-Jacques Rousseau, La transparence et l'obstacle, suivi de + Sept Études sur Rousseau*, Paris, Gallimard, 1971, p. 120 : * L'exaltation de la fete collective a la même structure que la volonté générale du Contrat social .

13. KOSELLECK, op. cit., p. 83. 
gation de l'homme envers lui-même *14, un idéal de formation suffit-il? Entre le droit et la sensibilité, quel art prévaut $^{15}$ ? Faut-il rationaliser le sentiment ou, au contraire, rendre la raison sensible? Avant que Kant ne publie sa Critique du jugement, qui situe les jugements esthétique et téléologique avec le jugement entre entendement et raison, c'est-à-dire entre théorie et pratique, le texte de Rousseau ne pouvait se prévaloir d'une position transcendantale, dont celui de Schiller se prémunissait. La conciliation des législations du cour de l'homme et de son esprit demeurait en quelque sorte dans une zone d'ombre précritique. Le débat que je tente d'établir entre eux engage déjà la mise en cause

14. Je me réfère ici au chapitre : \&a Nouvelle Hélö̈se de Rousseau et le Wertber de Goethe à l'intérieur du changement d'horizon entre le siècle des Lumières et l'Idéalisme allemand*, qui se trouve aux pages 276-354 de l'ouvrage de H.R. JAUSS, Pour une berméneutique littéraire, tr. fr. Maurice Jacob, choix d'études publiées dans Ästbetische Erfabrung und literariscbe Hermeneutik, Francfortsur-le-Main, Éditions Suhrkamp, 1982 ; Paris, Gallimard, 1988. Ici, p. 307.

15. La lecture que donnent Cassirer (op. cit., p. 35), Starobinsky (op. cit., p. 47-48) et meme Jauss (op. cit., p. 307-308), de l'interprétation de Rousseau par Kant dans ses "Conjectures sur les débuts de l'histoire humaine", (E. KANT, La philosophie de l'bistoire, tr. fr. S. Piobetta, coll. Médiations, Paris, Denoël, 1947 , p. 110-128) (*Mutbmasslicber Anfang derMenscbengescbicbte *, Ak, Bd 8), me semble se livrer à un rapprochement un peu rapide de l'art et de l'institution juridique. Alors que Kant résoud le dilemme de Rousseau, cherchant "comment la civilisation doit progresser pour développer les dispositions de l'humanité en tant qu'espèce morale », en affirmant que la contradiction entre ces dispositions et les progrès de la culture s'efface au moment où «l'art, atteignant sa perfection * devient " de nouveau nature *, je ferai deux objections à ce rapprochement. Pour Kant, le mot * art * signifie ici technique, c'est-à-dire des règles qui, reposant sur des concepts, comptent comme des corollaires de la philosophie théorique (cf. E. KANT, Critique de la faculté de juger, tr. fr. A. Philonenko, Paris, Vrin, 1984, Introduction, I, p. 22). L'art, en tant que production selon des règles qui ne peuvent être déterminées conceptuellement, n'est donc pas cet art que Kant appelle adroit civil * et qui permet à la culture de redevenir nature, même en comprenant art et nature au sens général de production d'après des règles. Seconde objection, chez Kant, le beau est destiné à symboliser le bien. Alors, comment pourrait-il etre cet art qui conjecturalement consiste à donner une conformité extérieure, celle de la loi universelle, a la liberté de chaque homme, s'il n'est que dans un rapport d'analogie avec cette idée de liberté qui est la seule a avoir des manifestations réelles, selon la Critique de la raison pure ? Même un idéal de formation doit posséder quelque force de conviction, qui ne peut reposer ni sur l'art politique ni sur la création artistique. Par ailleurs, pour Kant, c'est la décence qui est le « fondement réel de toute vraie sociabilité * (Kant, *Conjectures *, p. 115). Ce penchant * provoque chez autrui de la considération à notre égard pour nos bonnes manières (en masquant ce qui pourrait inciter au mépris) - (id.). C'est donc un sentiment lié à une valeur, estime ou mépris, qui préside au souci de l'apparence, que Rousseau condamne évidemment, et non le *sentiment esthétique*, d'où *peut surgir*, selon Jauss (p. 308), le * sentiment moral . Pour expliquer comment la raison commande au plaisir esthétique comme elle rend légitime le désir et que l'entendement ordonne l'expérience d'une cuvre d'art comme il légalise la connaissance, il fallait que Kant pose, dans la Critique du jugement, la question de la légitimité de l'imagination, cette faculté dont l'art se cache derrière tout sentiment, mais art que Kant incorpore dans tout jugement. Le problème que renouvelle Jauss est donc au mieux celui de la finalité de l'expérience esthétique, plutôt que celui de son origine. Sa réponse est celle de la polyfonctionnalité. 
du projet des «Lumières* d'autonomiser le domaine esthétique, dont Kant consacre les apories dans la «Dialectique du jugement esthétique». Nul ne peut déterminer ce qui doit me plaire personnellement, mais tous seront d'accord pour me laisser faire valoir mon sentiment comme étant le leur aussi, car rien d'autre ne nous oblige que cet accord ${ }^{16}$. C'est cette prétention même qui fera toujours litige, le problème de l'efficience d'une certaine liberté, lequel se retrouve dans la position de Jauss. Rousseau, par le contraste de la vie genevoise aux illusions du théâtre, et Schiller, par l'efficience d'une scène permanente au sein d'une société dominée par le changement constant de ses conditions de vie, témoignent ainsi, et certes de façon antithétique, de la situation antagonique de la jouissance esthétique. L'esthétique de la réception, en invitant à lire l'«histoire de la littérature et des Beaux-Arts [comme] l'histoire de la servitude et de l'insubordination foncière de l'expérience esthétique ${ }^{17}$, revient elle-même aux sources de la question de la normativité de l'expérience esthétique.

"L'attitude de jouissance esthétique dont l'art implique la possibilité et qu'il provoque, soutient H.R. Jauss, est le fondement même de l'expérience esthétique " ${ }^{18}$. La défense du plaisir en art s'élève contre la suspicion dont il a été l'objet, art et philosophie s'accordant difficilement depu is la troisième Critique de Kant sur les potentialités de l'expérience esthétique ${ }^{19}$. Celui qui jouit d'une œuvre d'art ou d'un spectacle apparaît floué par le désir, son expérience est sans portée pratique et n'a pas de valeur cognitive. Sa jouissance paraît faire éclater le cadre normatif dans laquelle elle inscrit sa "négativité", alors même que son attitude envers les ouvres est dominée par leur caractère «affirmatif» tout-puissant. Conduite à renouveler la question de la "praxis esthétique", qui en tant qu'activité productive, réceptive et communicative a été à la base de tout $\operatorname{art}^{20}$, l'esthétique de la réception se base entre autres sur la présupposition suivante. Selon la position de Jauss, que je

16. KANT, E., Critique de la faculté de juger, \#57, p. 163.169

17. JAUSS, "Esthétique de la réception et communication littéraire ", loc. cit., p. 1127

18. JAUSS, «Petite apologie de la jouissance esthétique «, op. cit., p. 125

19. Aperçue d'abord comme une limite a la rationalité, les a priori de l'activite esthétique ne peuvent pour Kant être circonscrits qu'*une fois qu'a été cernée la rationalité du pratique et du cognitif*. Cf. Jean GRONDIN, Kant et le probleme de la pbilosopbie : l'a priori, Paris, Vrin, 1989, p. 106. En contrepartie, il me semble que le *plaisir désintéressé * de la Critique du jugement posera une limite à la sensibilité, dont toute théorie de l'art aura du mal à se départir.

20. JAUSS, H.R., Préface de Aestbetic Experience and Literacy Hermeneutics, tr Michael Shaw, Minneapolis, University of Minnesota Press, 1982, p. xovii 
retiens ici, l' "expérience première d'une cuvre d'art a lieu dans l'orientation vers son effet esthétique, dans une compréhension qui est plaisir, et un plaisir qui est cognitif "21. Du simple point de vue pratique, quand l'expérience esthétique acquiert-elle une fonction créatrice de normes? Autrement dit, l'attitude ou l'expérience esthétique est-elle par elle-même capable d'acquérir une signification pour l'action pratique? Jauss affirme qu'elle le peut de façon médiate, l'art étant produit et reçu dans la liberté ${ }^{22}$. Il faudra le débat entre Rousseau et Schiller pour dessiner les voies d'élucidation de ce problème. Je n'utiliserai cette conception de la jouissance esthétique que dans un sens restreint. Il s'agit de comprendre le jeu théâtral selon l'articulation de trois moments : produire, recevoir et communiquer, autour de cette jouissance afin de différencier, dans l'interdit du théâtre et l'apologie de la scène, de quelle manière leur demeure reliée la prétention permanente de l'expérience esthétique à la jouissance. Pour comprendre la compétence que nous propose la scène de transformer ce qu'elle présente à notre exploration en "sources de bonheur» comme le veut Schiller (S., p. 821), il faut saisir d'abord pourquoi Rousseau ne peut séparer l'efficience du beau esthétique de celle du beau moral.

Ainsi la manière de l'un et de l'autre de lier politique, morale et esthétique me conduit à axer mon examen des principes que je viens d'exposer autour de deux questions. D'abord, peut-on considérer le théâtre comme une instance de représentation où seraient révélées les voies par lesquelles une société délibère en vue de se donner des institutions? C'est en quelque sorte la question que Schiller adresse à Rousseau, cherchant à faire valoir contre les talents du législateur du Contrat Social les capacités de médiation de la scène dans le monde de la vie. Ensuite, la mise en œuvre des effets propres à cette instance relève-t-elle de la jouissance esthétique? Cette question posée à Schiller, que mon recours à la conception de Jauss me permet de prêter à Rousseau, vise à repérer un moyen de convertir, au bénéfice de l'authenticité de ses plaisirs, l'incapacité présumée par Rousseau lui-même d'une société à voir le bien. À la recherche de la manière dont les ouvres d'art attirent et retiennent leur public ou sont rejetées par lui, la perspective jaussienne d'une interaction entre une scène et son public, entre la manière d'agir d'une ouvre d'art et celle de la

21. JAUSS, idem, p. xxix.

22. JAUSS, idem, p. xoxix-xl. Voir aussi pour cette médiation, la * Réponse à Claude Piché de Jauss, in Texte, 3, 1984, p. 193-201. 
recevoir, permettra, je l'espère, de discerner pourquoi cette conversion relève toujours d'une règle que l'œuvre d'art écrit dans sa réception même. C'est la fonction de l'art de se soustraire à toute hétéronomie, ce qu'illustre l'histoire de la fonction du théâtre.

\section{La Lettre à d'Alembert sur les spectacles}

\section{1) L'article * Genève * du point de vue de Rousseau}

D'Alembert proposait à Genève, cette "petite république" encore au seuil des "Lumières", d'établir un théâtre en ses murs comme c'était partout la tendance générale ${ }^{23}$. La promulgation de «lois sévères et bien exécutées sur la conduite des comédiens" permettrait à celle-ci d'avoir à la fois "des spectacles et des mœurs» et de jouir de l'avantage des uns et des autres ${ }^{24}$. La "réforme" de l'Europe est accessible à ceux qui veulent propager les "lumières" nouvelles $^{25}$. En effet, selon l'encyclopédiste, Genève ne désapprouve pas les spectacles en eux-mêmes, elle craint plutôt «le goût de parure, de dissipation et de libertinage que les troupes de comédiens répandent parmi la jeunesse ${ }^{26}$. Mais pour Rousseau, la proposition de d'Alembert ne résout rien à la question de la civilisation et de ses maux. Genève n'étant pas encore atteinte par la dissolution de la "vérité des rapports humains" 27 , il y va de sa protection. Allant même au devant des intentions de Rousseau, Genève répondit aux Encyclopédistes par la voie de ses autorités spirituelles. L'avis des pasteurs y ayant force de loi, elle n'accueillit ni troupe de théâtre ni théâtre en ses murs ${ }^{28}$.

Plaidant contre l'esprit général du siècle, Rousseau établit aux premières pages de sa Lettre une exigence dont la portée est décisive pour l'examen de ma première question. "Quand un homme ne peut croire ce qu'il trouve absurde, écrit-il, ce n'est pas sa faute, c'est celle de sa raison" ( $L$. d'A., p. 152). La raison, doit-on comprendre, a des bornes. Quelle

23. D'Alembert, Jean Le Rond, Article "Genève ", in Encyclopédie ou Dictionnaire raisonné des sciences, des arts et des métiers, tome septième, Paris, Briasson, David l'aîné, Le Breton, Durand, 1757, p. 573-578 de l'édition de 1963. Le paragraphe sur le théatre correspond à un huitième de tout l'article, p. 576-577.

24. Idem, p. 576.

25. Idem, p. 577.

26. Idem.

27. STAROBINSKI, Jean, Le Remède dans le mal, Critique et légitimation de l'artifice a l'age des Lumieres, Paris, Gallimard, 1988, p. 168.

28. Cf. Genève, d'Alembert, Rousseau, p. 354-355 de la présentation de Varloor. 
base alors est, aux yeux des hommes, la plus solide pour établir des institutions? Est-ce affaire de raison ou de sentiment? Rousseau affirme que la "raison humaine n'a pas de mesure commune bien déterminée, et qu'il est injuste à tout homme de donner la sienne pour règle à celle des autres * (L. d'A., p. 152, note). Il y a des principes communs, une évidence commune, mais chacun a sa propre raison qui le détermine. Il ne s'ensuit pas cependant que le sentiment de cette autonomie mène au scepticisme. Le sens du mot sentiment provient ici de l'identification, que fait Rousseau dans l'Emile, du sentiment et de l'acte de la conscience: "Les actes de la conscience ne sont pas des jugements, mais des sentiments ${ }^{29}$. Cette identification, Cassirer la considère comme le dernier mot de la doctrine du sentiment de Rousseau. Citoyen de deux mondes, du bonheur humain et de l'inconditionnelle loi, le sentiment "englobe l'activité pure du jugement, de l'évaluation et de la prise de position ${ }^{30}$. Exclu pour sa passivité du fondement de la morale, le sentiment quelqu'il soit n'en doit pas moins être protégé contre les préjugés et l'arbitraire des dogmatismes.

En découvrant la naturalité active du sentiment et en l'opposant à la distanciation de "l'état de réflexion * comme à "un état contre nature ${ }^{31}$, Rousseau répond aux visées de l'encyclopédiste par le paradoxe. La supériorité de la conscience morale sur une raison hypertrophiée, voire déhumanisée, est possible à la condition que raison et sentiment collaborent aux institutions de la liberté, jusqu'à ce que leurs deux activités s'identifient dans la conscience. Juridiquement, le projet des "Lumières" est trop ambitieux pour faire droit à la particularité d'un peuple libre de ses occupations et de ses plaisirs. Pour décider de l'efficience d'une fonction esthétique dans la vie des hommes, il importe d'écarter d'abord les obstacles qui bloquent l'accès aux vérités pratiques qui les concernent. Le théâtre, public, permanent, ne peut, selon Rousseau, leur donner accès à la même immédiateté que celle que procure la conscience individuelle ${ }^{32}$.

29. ROUSSEAU, Émile, p. 377

30. CASSIRER, op. cit., p. 102.

31. ROUSSEAU, J.J., *De l'inégalité parmi les hommes*, in Du contrat social, Discours sur les sciences et les arts, Discours sur l'origine de l'inégalité parmi les bommes, Paris, U.G.E. (coll. 10/18), 1963, p. 261. Ci-après CS.

32. Comme il suffit de le faire pour saisir * dans toute sa clarté et dans la certitude immédiatement convaincante qui lui est propre * ce que Cassirer appelle l'«intuition * des vrais principes du domaine éthique, op. cit., p. 96. 
Luttant contre toute dissociation, Rousseau cherche les moyens de fondre dans la vie collective l'exigence de cet accès, garant de la propre préservation de toute communauté. En formulant les questions laissées en plan par d'Alembert: si les spectacles *peuvent s'allier avec les mours, si l'austérité républicaine les peut comporter, s'il faut les souffrir dans une petite ville" (L. d'A., p. 157), il indique déjà la portée qu'il donne à l'impératif du sentiment. Sa Lettre de 1758 vient donner une nouvelle assise à "l'antinomie entre le bien moral et la civilisation sur laquelle il base sa doctrine des arts», selon M. Moffat ${ }^{33}$. En écartant les arguments tout faits des polémiques de son siècle sur l'art et la moralité publique, elle évite de répéter la critique des deux Discours. Avec le mûrissement de la Nouvelle Héloïse, publiée en 1761 mais achevée la même année, le résultat de la critique de la civilisation se trouve transposé dans la nouvelle sensibilité in térieure chargée de s'imposer contre le goût régnant. Avec les souvenirs de sa jeunesse genevoise : l'idée de la communauté des Montagnons, l'image de la fête à Saint-Gervais, Rousseau esquisse la nouvelle expérience de l'«homme sensible», qui, selon l'interprétation de Jauss, "promet de faire retrouver le naturel perdu dans un sentiment de soi autonome»34. La liberté de la conscience est-elle conciliable avec la réalité des désirs et des besoins des hommes ? En effet, Rousseau écrit : *Je n'aime point qu'on ait besoin d'attacher incessamment son cœur sur la scène, comme s'il était mal à son aise au-dedans de nous" (L. d'A., p. 158). Je crois possible de voir dans cet aveu le leitmotiv profond de sa Lettre. Il lui reste à montrer comment l'accord du sentiment personnel et du droit public est réalisable.

Mais Rousseau demeure l'auteur de Narcisse, sa pièce. Soupçonné d'auto-contradiction par ses contemporains, la solution qu'il préconisait dans la Préface de Narcisse ( $L$. d'A., p. 133), a encore du prix à ses yeux. C'est la métaphore du remède dans le mal, laquelle, écrit Starobinski, «assimile le changement social au déclin et aux atteintes que subit un corps vivant ${ }^{35}$. Mais qui connaît mieux les «hommes tels qu'ils sont», du thérapeute ou du législateur? Avec l' «indifférence» des spectacles, Rousseau m'apparaît jouer avec ses propres interdits. La solution homéopathique de la Préface visait à prévenir une plus grande corruption, partout où elle existe, en procurant à ceux dont ce sont les plaisirs ces

33. MOFFAT, M., op. cit., p. 65

34. JAUSS, Pour une berméneutique littéraive, p. 294

35. STAROBINSKI, Le Remede dans le mal, p. 174 . 
plaisirs mêmes comme ils sont. La considération du cas particulier que représente Genève détermine, en fait, dans sa Lettre à d'Alembert sur les spectacles, le registre des oppositions aux effets du théâtre. Une dénonciation péremptoire du "simulacre public " de vertu que laissent les mêmes causes qui ont détruite celle-ci ne suffit plus. Il faut à Rousseau un «critère qui authentifie la civilisation ${ }^{36}$.

\section{2) Moyens d'assurer l'authenticité des plaisirs}

Le théâtre moderne, en gagnant sur les autorités séculaires son autonomie, s'était arrogé la prétention millénaire à être un moyen public de jugement moral. Telle était son arrogance, selon Jauss ${ }^{37}$. Pour Rousseau, cette prétention est sans fondement et son caractère illusoire rend caduque l'opinion courante qui veut que le théâtre, si plaisant qu'il paraisse, soit une "école de mours » 38 . Il est vain de vouloir rapprocher l'illusion de la réalité en demandant, comme le fera Diderot, que les personnages du drame bourgeois soient des bourgeois 39 . La question du théâtre doit plutôt porter sur l'originalité du droit de présenter des pièces dans un lieu public permanent.

\section{a) L' «indifférence " des spectacles}

Il est évident que Rousseau n'admettra jamais qu'on puisse, pour régler la question des effets des représentations théâtrales, avoir recours à des lois régissant la conduite des comédiens. Les comédiens sont au service de la comédie, affirme-t-il, et le seul but des auteurs est de plaire. Rousseau sait bien que «le jeu du comédien (...) ne prétend pas qu'on le prenne en effet pour la personne qu'il représente" ( $L$. d'A., p. 239). Mais défendre aux comédiens d'exercer leur profession, ce n'est pas leur interdire de défendre leur rôle. Les effets de la comédie n'en auraient pas moins lieu, nonobstant les leçons de mœurs qu'on prodiguerait aux comédiens. Comédiens et comédiennes sont invités sans plus à abandonner leur métier qui fait bon commerce de la tromperie. Pour Rousseau, il n'y a que «trois

36. Idem, p. 19. Starobinski y analyse les variantes du mot.

37. JAUSS, Aestbetic Experience and Literary Hermeneutics, p. 39

38. D'ALEMBERT, -Lettre à Jean-Jacques Rousseau, citojen de Genève *, ( $L$ d'A, p. 361), réplique que ce qui règle le jugement du public, *c'est uniquement le degre de plaisir ou de lumière qu'on lui a donne*.

39. Le théatre dramatique, selon Diderot, doit peindre des conditions sociales plus que des caractères. Il faut mettre en scène des personnages du meme milieu bourgeois que la majorité des spectateurs. Cf. Robert NIKLAUS, «Diderot et Rousseau. Pour ou contre le theatre $*$, in Diderot Studies, IV, 1963, p. 175. 
sortes d'instruments, à l'aide desquels on puisse agir sur les moeurs d'un peuple; savoir, la force des lois; l'empire de l'opinion, et l'attrait du plaisir" ( $L . d^{\prime} A$., p. 165). Et d'emblée, il a exclu le théâtre, parce que, à l'évidence, il *n'y a que la raison qui ne soit bonne à rien sur la scène" (L. d'A., p. 161). Comment, en effet, prendre plaisir à considérer les actions d'un homme raisonnable? Corollaire de cette exclusion, l'action des trois instruments présumés est nulle sur le théâtre lui-même. La contrainte des lois ferait du divertissement théatral une peine et non un amusement. Quant à l'opinion, au lieu d'en imposer une au public, le théâtre la reçoit plutôt de lui. Enfin, le plaisir du théâtre n'a pas d'autre attrait que de nous y ramener ( $L$. d'A., p. 165-166).

Le choix de ces trois sortes d'instruments dirigera mon examen de la différence sur laquelle Rousseau invite à statuer ( $L$. $d$ 'A., p. 220). Les principes généraux de son interdit du théâtre me semblent en effet gouvernés par l'office bien particulier qu'il assigne au législateur dans $D u$ contrat social. Il vient d'établir sur l'exemple de la communauté isolée des Montagnons la nocivité des spectacles, prouvant que, même s'ils n'étaient pas mauvais en eux-mêmes, "on aurait toujours à chercher s'ils ne le deviendraient pas à l'égard du peuple auquel on les destine" (id.). La différence entre les villes, grandes et petites, lui fait demander si les spectacles, mauvais pour un peuple bon, bons pour un peuple mauvais, ne pourraient pas «rester indifférents à tous». La métaphore du remède dans le mal est vite épuisée si le mal ne fournit plus de remède. Une société a moins besoin d'un thérapeute que d'un législateur. Le laisser-aller ou le laisser faire dans le domaine esthétique n'est-il pas lui-même préférable à l'action politique telle qu'elle a cours? Le moyen de resserrer les liens entre les hommes ne sera pas "l'intérêt personnel ", qui les met plutôt «dans une dépendance mutuelle » 40 .

\section{b) L'anticipation du recours au législateur}

L'ordre politique peut certes légiférer sur les théâtres et sur les hommes, auteurs ou comédiens. Comment s'assurer toutefois qu'il réponde, par l'institution des théâtres, aux désirs du peuple ou au besoin réel de divertissements d'un peuple? Non pas, dit Rousseau, "qu'avec du sens et de l'équité tout homme ne pût très bien trouver de lui-même [les lois] qui, bien observées, seraient les plus utiles à la société " ( $L . d^{\prime} A$. , p. 222), mais cela ne saurait suffire sans la connaissance de certains rapports, des véritables rapports

40. ROUSSEAU, «Préface du Narcisse (1753), textes annexes à la $L d^{\prime} A$, p. 129 
des choses. L'expérience du théâtre a lieu dans la distanciation entre une scène et un public, laquelle n'offre en représentation qu'une image bien lointaine des liens intimes qui unissent les membres d'une société, d'un peuple ou d'une communauté. Rousseau réitère sa méfiance envers tout ce qui détourne de l'immédiateté naturelle dans laquelle les hommes évoluaient jadis en toute sûreté avec, d'après le Premier Discours, "la facilité de se pénétrer réciproquement" (L. d'A., p. 47).

Deux raisons expliquent chez Rousseau l'exclusion de tout rapport entre théâtre et législation, sans exclure toutefois une certaine tension entre elles. La première est exprimée au chapitre "De la loi" de Du contrat social, qui précède immédiatement celui «Du législateur». La seconde tient à l'impossibilité de déléguer des représentants. Rousseau écrit que, faute de sanction naturelle, il faut "des conventions et des lois pour unir les droits aux devoirs et ramener la justice à son objet» $(C S, \mathrm{II}, \mathrm{VI}, \mathrm{p} .81)$. Le théâtre a déjà été exclu de la sphère d'influence des lois, qui transformeraient en obligation ce qui doit être un divertissement. Il a, en effet, " ses règles, ses maximes, sa morale à part, ainsi que son langage et ses vêtements» (L. d'A., p. 170). À quoi sert tout cela, demande Rousseau. Â «reléguer à jamais sur la scène" "tous les devoirs de l'homme" (id.), répond-il. Le théâtre pourtant semble appartenir à la vie des peuples, à la conscience qu'ils prennent d'eux-mêmes; il n'est régi que par ces conventions qui "règlent les conditions de la société * (CS, p. 81). Les jeux d'ombres et de lumière particuliers aux représentations théâtrales ne sont-ils pas cependant contraires au devoir général de transparence? Le droit de représentation serait un droit original, si, soutient Rousseau, un peuple jouissait de la compétence d'approprier celle-ci à lui-même. C'est ainsi qu'il affirme ne voir qu'«un remède à tant d'inconvénients»: pour s'approprier les drames de leur théâtre, les Genevois n'ont qu'à les composer eux-mêmes (L. d'A., p. 290).

L'appropriation qu'il propose ici dépasse à nouveau la solution du remède dans le mal. Ia Lettre sur les spectacles vise à remplacer le théâtre par des institutions récréatives : fêtes en plein air l'été, bals communaux l'hiver, pour ainsi dire propres à la société future du Contrat social. Une certaine reconnaissance se fait jour ici du concours d'une fonction esthétique au bien général. Ce ne serait finalement qu'une question de convention que de choisir les moyens d'agir sur les mœurs en corrigeant l'opinion que les parti- 
culiers ont de leur vie commune, actuelle et passée. L'affinité des goûts et des mœurs plaide en ce sens. À un certain état du goût correspond toujours un certain état des mours, établit Rousseau (L. d'A., p. 162, note). Les lois et les mœurs sont en un double rapport d'influence. Les lois n'agissent sur les mours que parce qu'elles en tirent leur force et que les mœurs * leur rendent cette même force " (L.d'A., p. 222). Seules sont observées les lois qui ne changent pas "la nature des choses", mais "ne font que la suivre" (L.d'A., p. 238). Cependant l'appartenance du théâtre à la fiction l'éloigne de tout lien véritable avec l'authentique sentiment de soi qui correspond aux vérités pratiques d'une société. «Plus j’y réfléchis, soutient Rousseau, et plus je trouve que tout ce qu'on met en représentation au théâtre, on ne l'approche pas de nous, on l'en éloigne» (L. d'A., p. 170). La présence d'un dispositif scénique dans la cité est à elle seule intolérable ${ }^{41}$. Et il appuie sa condamnation sur la réflexion suivante: «l'effet qui renforce le bien et le mal, étant tiré de l'esprit des pièces, est sujet comme elles à mille modifications qui le réduisent presque à rien; au lieu que celui qui change le bien en mal et le mal en bien, résultant de l'existence même du spectacle, est un effet constant, réel, qui revient tous les jours et doit l'emporter à la fin» $(L . d$ 'A., p. 221).

Droits et devoirs sont donc affaire de justice et non de représentation théâtrale, tant que la condition de leur appropriation n'est pas remplie. Voilà d'où naît la nécessité du législateur, selon Du contrat social. « De lu i-même, explique Rousseau, le peuple veut toujours le bien, mais de lui-même il ne le voit pas toujours" (CS., p. 83). Éclairer ceux qui se fusionnent dans la volonté générale apparaît nécessaire, quand il s'agit de "faire voir" au souverain, au peuple assemblé, au moment où il donne à l'institution, au pacte social qui le détermine, la faculté d'agir, «les objets tels qu'ils sont, - et ajoute Rousseau -, quelquefois tels qu'ils doivent lui paraître" (id., p. 84). La présence du législateur, qu'anticipe sa Lettre à d'Alembert, apparaît encore plus évidente, quand il s'agit de faire jouer une appropriation bien particulière : celle du code de lois au peuple et de la loi à son objet. Pour cela, deux mesures s'offrent au législateur : celle de la force des lois dont le code est à écrire et celle des vices qu'elles sont destinées à réprimer. Après avoir compa-

41. Il en est des theatres comme des tréteaux qui, dans la rue, à la foire, servent a l'improvisation d'une représentation quelle qu'elle soit, sauf peut-être les montres de marionnettes. 
ré ces forces et trouvé que la première surpasse la deuxième, le législateur sera assuré de l'exécution de la loi. Le législateur peut-il cependant agir sur quelque chose dont il ignore comment il agit, s'interrogera Schiller? La convention souveraine du théâtre, la fiction, ne lui échappe-t-elle pas? Sa tâche n'a d'autre but, en effet, que de donner à un peuple les meilleures lois qu'il puisse supporter dans une situation donnée, en appropriant «tellement ce code au peuple pour lequel il est fait, et aux choses sur lesquelles il statue, que son exécution s'ensuive du seul concours de ces convenances* (L.d'A., p. 222). La scène pourra-t-elle s'approprier ce concours de convenances?

Au théâtre, admet Rousseau, ni l'auteur ni le jeu des comédiens ni les artifices de la scène ne prétendent faire voir ou apercevoir les choses dans leurs rapports "véritables» $\left(L . d^{\prime} A .\right.$, p. 171$)$. C'est qu'il l'en croit incapable, mais un même rapport d'exclusion vaut, comme à son insu, pour le législateur lui-même. Le modèle législatif du Contrat social n'a pas pour but premier de donner à chaque membre d'une communauté la compétence d'approprier la fiction à la réalité, selon les «convenances» recherchées. Le théâtre et le législateur ne laissent pas non plus libre cours au sentiment pratique qu'a un peuple de ce qui lui convient dans ce qui le détermine. Le lien entre un théâtre et son public comme le lien entre un peuple et le législateur de ce peuple sont pareillement exclusifs, l'un de la délibération, l'autre de la représentation. L'interdit du théâtre correspond à l'impossibilité pour un peuple de déléguer à l'assemblée générale des représentants pour délibérer à sa place.

La seconde raison d'exclure le théâtre de la réalisation du bonheur relève, elle aussi, de la préservation de l'originalité du sentiment autonome de soi. L'accès à cette vérité fondamentale ne se réalise que dans un mouvement de conversion des libertés. Le Contrat social en donne la formulation: «quand le peuple statue sur tout le peuple, il ne considère que lui-même; et s'il se forme alors un rapport, c'est de l'objet entier sous un point de vue à l'objet entier sous un autre point de vue, sans aucune division du tout» (CS., p. 82). C'est la possibilité de ce rapport qui est génératrice de la tension au sein des exclusions de Rousseau. Il s'agit d'arriver à la fusion des intérêts particuliers dans la volonté générale, mais un dédoublement, comme un effet simultané, empêche la réalisation de cette conversion. Une distanciation semblable à une réflexion et à celle pour laquelle Rousseau condamne le théâtre s'insinue dans l'as- 
semblée générale. Les particuliers qui composent un peuple manquent certes de perspective, mais l'immédiateté dans laquelle ils se trouvent leur interdit de se représenter euxmêmes dans ce rapport.

Rousseau joue, comme je le mentionnais, avec ses interdits. La distanciation concomitante au rapport de réflexion d'un peuple ou d'un individu sur lui-même n'est pas le correctif adéquat à sa cécité passagère. Pour qu'une loi devienne représentation commune, le législateur doit la "traduire dans la langue du peuple», écrit Rousseau (CS., II, VII, p. 87), la lui présenter comme le résultat de sa propre délibération. Sauvegarde du domaine des conventions, des us et coutumes, le législateur se trouve situé à la périphérie du cercle de délibération d'un peuple assemblé. Rousseau interdit paradoxalement de recourir à lui pour assurer l'authenticité des plaisirs d'une communauté. Les rapports intérieurs d'une société entre le sentiment qu'elle a d'elle-même et la volonté qu'elle peut avoir de se soumettre à sa propre loi lui échappent, faute d'avoir la compétence d'établir une instance de représentation, théâtrale ou politique, convenant à ce qu'elle est. Mais, ultime paradoxe de la position de Rousseau, l'interdit de la représentation théâtrale ne rend-il pas, sans cette double médiation, tout à fait inefficace l'opinion publique, le seul moyen pertinent d'agir sur les cœurs comme sur les mœurs?

\section{c) passage à l'esthétique}

La possibilité d'une conversion "grise» de l'opinion, grise parce qu'un peuple ne voit pas toujours clairement le bien, correspondrait à une convention particulière. Elle plaiderait ainsi en faveur d'un accord aussi spontané qu'insoupçonné. La compétence d'une société à s'interdire le plaisir du théâtre ne peut s'expliquer que dans un cadre normatif, à moins que, transgressant l'interdit, le théâtre ne confonde l'opinion publique par sa propre normativité. Pour Rousseau, "ni la raison, ni la vertu, ni les lois ne vaincront l'opinion publique, tant qu'on ne trouvera pas l'art de la changer" ( $L$. d'A., p. 226). La volonté générale veut-elle apprendre cet art, il répond "que c'est par l'opinion publique", c'est-à-dire "nulle espèce de moyens coactifs» (L. d'A., p. 223). L'art de changer l'opinion publique répond au même mouvement d'exclusion contre lequel une convention particulière doit être cherchée là où la volonté générale ne peut se représenter la possibilité de statuer sur le bien. Mais le public peut-il apprendre l'art du théâtre, qui 
comme l'art de changer l'opinion publique, "ne tient point à la violence» (L.d'A., p. 232)?

La Lettre à d'Alembert sur les spectacles prolonge les arguments classiques contre la mimèsis; la promesse de l'imitation théâtrale : corriger les mœurs en les chargeant ${ }^{42}$, est fallacieuse, stipule Rousseau. Comment alors convenir entre particuliers de l'»indifférence * des spectacles? Comment appliquer la conversion *grise * à l'opinion publique? Il s'en faudrait de peu qu'échappant à la "duplicité" de la représentation - attacher son cour sur la scène n'est-ce pas accepter sans condition ce qui s'y présente? - l'opinion publique soit sauvegardée de tout effet de suggestion ${ }^{43}$. Rousseau refuse de considérer comme facteur d'institution d'une société ou de formation d'un peuple autonome l'expérience esthétique. Qu'elle soit hors la loi ne prouve pas ipso facto qu'elle soit libre. Une société, si «éclairée » qu'elle le devienne, ne possèdera jamais totalement la compétence d'approprier ses institutions à ce qu'elle a le sentiment d'être et de devoir être. Toutefois elle ne peut pas quitter l'immédiateté de ce qu'elle est. La seule convention générale que les particuliers peuvent considérer comme étant appropriée à leur nature et à la constitution de leur société, c'est pour Rousseau l'absence de contrainte. Tout le reste doit appartenir à la volonté générale, qui force à être libre. Le chapitre De la censure du Contrat social en est l'illustration paradoxale. Rousseau y écrit que si «la loi ne règle pas les mœurs, c'est la législation qui les fait naître" (CS., p. 175). Comment les changer, sans changer la constitution de toute la société? La situation antagonique où se trouve les jouissances passées d'un peuple avec sa liberté future ne plaide-t-elle pas en faveur des possibilités de la création artistique? Le théâtre, à l'instar de la conception de Jauss, ne peut-il communiquer ces connaissances "pratiques" dont Schiller le croit chargé ? La scène ne pourra-t-elle apprendre ce que signifie vivre dans l'absence de contrainte en communiquant dans son propre langage un autre sens du mot "appropriation": non plus ce qui est contrôlé par un code sous le signe d'une maîtrise des forces antagoniques, mais ce que chacun a fait sien pour l'avoir goûté et partagé avec d'autres, ce qu'on apprécie pour soi-même dans la découverte où conduit le plaisir de l'autre ${ }^{44}$ ? Sans

42. Nous dirions en accuser les traits pour en faire ressortir l'odieux ou le ridicule, ce qui n'est pas étranger à la décence de Kant, cf. plus haut, note 15.

43. Pour Jauss, Rousseau anticipe ici le caractère manipulateur de la culture officielle, op. cit. , p. 39-40.

44. JAUSS, «La jouissance esthétique*, loc. cil., p. 270 
cette expérience, aucun plaisir particulier ne semble en vérité publiquement «approprié».

\section{3) Rousseau et les institutions du * sentiment *}

L'ouverture du Discours sur les sciences et les arts subvertissait la question de l'Académie de Dijon : si le rétablissement des sciences et des arts a contribué à épurer ou *à corrompre* les moeurs. Un contre-pied théorique semblable justifie par avance la condamnation du spectacle théâtral de la Lettre à d'Alembert. Le théâtre, au même titre que les sciences et les arts est un fruit de l'oisiveté. La question de savoir ce que sont les représen tations théâtrales est depuis toujours déjà subordonnée à celle de la moralité des institutions pour le divertissement en société. Les spectacles étant destinés au "peuple", Rousseau demande, narquoisement, si ces représentations ne devraient pas lui être appropriées. À l'encontre de cette prétention à la soumettre à un cadre normatif, l'œuvre théâtrale n'est manifestement pas l'œuvre d'un législateur. Les objections par lesquelles Rousseau interdit l'exercice concomitant de la représentation et de la délibération valent aussi pour le théâtre, dans l'impossibilité où il se trouve de changer l'opinion dont il origine. La présence d'une convention particulière, la possibilité d'une conversion "grise* de l'opinion, me semblent cependant témoigner d'un envers à cet interdit du théâtre qui originerait, lui, du sentiment. Cet envers relèverait de la double participation de l'expérience esthétique à la liberté, pour laquelle aucune formation ne paraît nécessaire ${ }^{45}$. Le problème de l'efficience de cette liberté se retrouve à l'avant-plan. Le critère quasi-juridique de la Lettre à d'Alembert a laissé Rousseau pour ainsi dire muet sur cette question. Je me propose de considérer quelles en sont les présuppositions en vue de saisir pourquoi, en préservant une société contre les préjugés et les intérêts qui contraignent l'opinion, il la condamne apparemment à ne jamais connaître dans ses divertissements l'attrait du plaisir?

\section{a) Les présuppositions de la Lettre à d'Alembert}

Deux thèmes centraux de la pensée de Rousseau alimentent l'argumentation de sa Lettre. Le premier, c'est «que l'homme est né bon» (L. d'A., p. 167). Contre les prétentions didactiques du théâtre dramatique bourgeois, Rousseau soutient que «la source de l'intérêt qui nous

45. Cf. plus haut, p. 109. 
attache à ce qui est honnête et nous inspire de l'aversion pour le mal, est en nous et non dans les pièces». Que signifie pourtant notre attachement à l'imitation que propose le théâtre? S'agit-il ici de l'un de ces actes de la conscience que Rousseau identifie à un sentiment, et qui pourtant est identique à un jugement? «L'amour du beau, explique-t-il, est un sentiment aussi naturel au cœur humain que l'amour de soi-même" (L. d'A., p. 167). Pour comprendre comment a lieu cette double identification, la référence à l'autonomie de la conscience comme au fondement de l'éthique ne suffit plus. Il s'agit plutôt d'interroger à nouveau le sentiment en vue de découvrir l'originalité de l'expérience esthétique. Certes, la Lettre sur les spectacles représente un moment intermédiaire, entre la critique des "mœurs" civilisées des deux Discours et l'idéal de formation de l'Émile et de $D u$ contrat social, dans la solution de la question "du but à fixer à l'homme" 46 . Dans l'interprétation de Jauss d'un changement d'horizon entre Rousseau et Schiller, la perspective de regagner la chance du bonheur relève évidemment du recouvrement par l'homme, face à la dissociation de son existence comme homme civil, de la totalité perdue de l'homme naturel. En somme, comme l'écrit Cassirer, l'institution civile fondée en droit a pour fonction première d'apprendre à ceux à qui elle s'adresse à reconnaître dans la loi "la nécessité qui la motive", de leur apprendre à se plier à la loi commune comme on obéit à la nature ${ }^{47}$. L'idéalisme allemand n'aura pas d'autre programme ${ }^{48}$.

L'originalité de Rousseau a toujours été de plaider en faveur d'une nouvelle sensibilité propre au domaine pratique. L'idéal de formation, de rationalisation du sentiment, emprunte la voie inverse de la sensibilisation de la raison. Cette possibilité se fait jour dans le commentaire de Rousseau par Kant, lequel convertit le statut anti-téléologique de la "faculté de se perfectionner" du deuxième Discours en son contraire ${ }^{49}$. La négation d'une finalité de la nature servait à Rousseau à la fois de principe d'explication de la dichotomie entre le progrès des sciences et des arts et la corruption des mœurs comme "l'œuvre même de l'homme", et d'argument pour exiger "la libération de la

46. JAUSS, Pour une berméneutique littéraire, p. 298.

47. CASSIRER, op. cit., p. 40.

48. Ce programme visera, selon Jauss, à résoudre * le conflit des deux aptitudes de

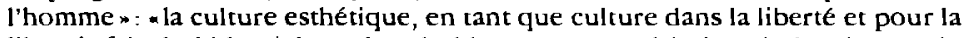
liberté, fait de l'identité perdue de l'homme naturel le but de l'action et du devenir de l'homme dans le monde civil ", Pour une berméneutique littéraire, p. 326

49. ROUSSEAU, „De l'inégalité parmi les hommes », Première partie, $C S$, p. 265 ssq 
nature oppressée " 50 . Mais Kant a préféré conjecturer sur la culture, sur le dessein de la nature laissé aux propres ressources des hommes, plutôt que sur l'état de nature. C'est à bon droit, affirme-t-il, que Rousseau a dénoncé la funeste inégalité qu'un développement anarchique, voire achronique, de la culture a introduite parmi les hommes, mais c'est une question plus difficile encore qui s'est posée à lui que celle de «savoir comment la civilisation doit progresser pour développer les dispositions de l'humanité en tant qu'espèce morale, conformément à leur destination " 51 .

La question de l' « indifférence" des spectacles fait apparaître dans la Lettre à d'Alembert un parallèle entre cette *indifférence" et l'efficience de la liberté. La sensibilité nouvelle qu'un peuple peut prendre de lui-même, en le convaincant de la nocivité des spectacles sur lui, doit lui permettre de sauvegarder sa propre nature en la transfigurant. La faculté de s'arracher aux contraintes de la nécessité de son espèce, l'œuvre de l'homme individuel, peut être restaurée dans l'art. Cet art, l'art politique, fait appel à la naturalité du sentiment presque autonome du beau moral, pour concilier l'in térêt personnel et le but de la société, la bonté du bien. La conservation de soi, qui rend l'homme indifférent aux autres, est mise en étroite correspondance avec la «répugnance à voir périr ou souffrir tout être sensible, et principalement nos semblables " 52 . En affirmant que ce sont là «deux principes antérieurs à la raison» 53 , Rousseau s'achemine vers la formulation de la loi de cette "conversion" de l'autre en soi par la médiation d'une fonction esthétique ${ }^{54}$. Exclu des principes de l'éthique, du règne rationnel de la loi civile, le sentiment esthétique est reconduit à sa nature, à la sensibilité. Jauss en relève la définition chez Rousseau : «Il y a une autre sensibilité que j'appelle active et morale qui n'est autre que la faculté d'attacher nos affections à des êtres qui nous sont étrangers " 55 . Rousseau, par contre, n'a-t-il pas rejeté cet attachement comme un malaise du cœur? Cette sensibilité n'est-elle pas contredite dans sa prétention par le second Discours qui déclare originellement responsable du prix qu'a acquis "l'estime publi-

50. JAUSS, op. cit., p. 300-301

51. KANT, "Conjectures..., p. 119-120

52. ROUSSEAU, *De l'inégalité parmi les hommes *, $C S$, p. 249

53. Idem.

54. But affectif de la société, la * sym-pathie * appartient, selon Cassirer, à la * bonté * originelle de l'homme comme a une tendance et a une destination fondamentales de la w volonte . Cassirer, op. cit., p. 89-92.

55. ROUSSEAU, Euvres complètes, La Pléiade, I, p. 805. Jauss, Pour une berméneutique littéraire, p. 297, note 3. 
que» la capacité qu'eurent les oisifs de devenir spectateurs 56 ? Quelle est donc la loi naturelle qui oblige le sentiment à se subordonner à la raison ?

Le second thème de l'argument anti-théâtral, Rousseau le formule ainsi : "L'homme est un" ( $L$. d'A., p. 159). La relativité des situations humaines exige en contrepartie de se livrer à un exercice de particularisation : «l'homme modifié par les religions, par les gouvernements, par les lois, par les coutumes, par les préjugés, par les climats, devient si différent de lui-même qu'il ne faut plus chercher parmi nous ce qui est bon aux hommes en général, mais ce qui leur est bon dans tel temps ou dans tel pays» (L. d'A., p. 158-159). Rousseau en a fait la mesure du rapport entre les spectacles et un peuple. Ce pragmatisme esthétique, sans la conversion "grise" de la sensibilité pratique qu'un peuple a de luimême, est-il réellement applicable au domaine esthétique? Est-ce encore l'office du législateur de décider quels spectacles destiner à tel peuple? L'une des formulations par lesquelles Jauss définit l'expérience de jouissance esthétique corrobore la difficulté de prescrire à tel organisme social le remède qui fait l'essence de sa maladie, en ce cas l'ordre du bonheur. Jauss, en effet, conçoit l'expérience esthétique comme « jouissance de soi-même dans la jouissance de l'autre " 57 . Personne ne peut quitter l'état d'homme, pour entrer dans un état de nature transfiguré, sans passer par la médiation de la culture. Celle-ci n'atteint sa perfection que quand l'art politique travaille à la moralisation du genre humain et que le mot art signifie formation. Mais, dans la perspective de Jauss, la découverte de l'altérité dans son propre plaisir doit pouvoir restituer à la "pratique» esthétique sa liberté de manœuvre dans le monde de la vie.

Revenant à la question initiale, Rousseau écrit que "pour juger s'il est à propos ou non d'établir un théâtre en quelque ville, il faut premièrement savoir si les mœurs y sont bonnes ou mauvaises" (L. d'A., p. 221). Comment juger de l'appropriation des spectacles à telle société, sans convertir le sens de ce mot? Participer et s'approprier correspondent sans doute au mouvement de l'ouverture de soi à l'autre dans lequel se propose la chose qui en est l'enjeu, comme au théâtre se propose dans le jeu théâtral le sens qu'il nous

56. Le chant et la danse devenant * l'amusement et plutot l'occupation des hommes et des femmes oisifs et attroupés *, chacun "commença à regarder les autres et à vouloir être regardé soi-meme *. Rousseau, « De l'inégalité parmi les hommes *, Seconde partie, CS, p. 299.

57. JAUSS, « La jouissance esthétique •, loc. cit., p. 270. 
appelle à vivre. C'est ici que l'absence de contrainte rejoint la liberté dans laquelle l'art est produit et reçu, dans laquelle il se communique. Quelle forme de communication Rousseau propose-t-il alors à la place du théâtre? La fête, mais n'est-ce pas sans avoir déterminé si le sentiment pour le beau, celui qu'engendre l'art, est aussi naturel à l'homme que l'amour de soi ? Rousseau envisage plutôt le problème du point de vue de la fin qu'arts et sciences servent. Le verdict du premier Discours est connu : "l'effet est certain, la dépravation réelle» ( $L$. d'A., p. 49). L'identification de la nature et de la culture, dans la perspective téléologique de Kant, n'est pas possible dans l'horizon juridique de Rousseau. Ne peut-on alors par des institutions relevant cette fois du «sentiment " contrer "cette faculté distinctive et presque illimitée", celle de se perfectionner, qui est "la source de tous les malheurs de l'homme " 58 ? Le second Discours, en ce sens, s'emploie à énoncer la loi naturelle qui convient le mieux à la constitution humaine: "pour qu'elle soit loi, il faut que la volon té de celui qu'elle oblige puisse s'y soumettre avec connaissance, mais (...) encore, pour qu'elle soit naturelle, qu'elle parle immédiatement par la voix de la nature "59. Malgré la double citoyenneté du sentiment, le sentiment qui porte l'homme à jouir des représentations de lui-même le jette hors de sa nature, le théâtre ne présentant jamais qu'un «tableau des passions humaines, dont l'original est dans tous les cœurs» (L. d'A., p. 160). Il n'y a pas d'institutions esthétiques qui, comme le théâtre, jouiraient de leur propre légalité. En d'autres termes, il n'existe pas pour Rousseau de loi naturelle en vertu de laquelle le théâtre puisse mettre en correspondance sentiment particu. lier et assentiment public.

À l'évidence, la raison n'est bonne à rien sur la scène. Le critère de l'utilité, sur lequel Rousseau se retourne, a toute validité, mais à la seule condition d'interdire au théâtre de représenter l'adhésion spontanée de chacun à la loi de tous. Comment juger ultimement de la qualité d'un spectacle eu égard au peuple auquel il est destiné, et de la moralité de tel spectacle eu égard à l' "état d'homme" dont il est soupçonné de divertir en tant qu'amusement, sinon en interrogeant Schiller sur l'efficience de la représentation théâtrale? La voie de la concrétisation de la promesse du plaisir esthétique croise le chemin du droit; entre eux se creuse une nouvelle faille dans la conciliation du sentiment

58. ROUSSEAU, « De l'inégalité parmi les hommes *, CS, p. 266

59. CS, p. 249. 
et de la raison. Le travail anéantit par son résultat la vocation à la liberté. Qui éduque? Qui instruit? Qui amuse? Qui réfléchit? Ces questions, que résume celle de la division du travail, dominent, dans les Souffrances du jeune Werther de Goethe, la réalisation de la liberté 60 . Nouvelle manière de ressentir et d'agir, entre la doctrine du sentiment de Rousseau et l'activité du sens esthétique chez Schiller, la souffrance vient tracer la figure des hommes dissociés, qui échappent à toute formation. Quelle ceuvre peut alors les rejoindre, si l'auteur, lui-même dissocié de celle-ci, n'est plus désormais "obligé de $[\ldots]$ donner un idéal moral ${ }^{61}$ ? Pourtant les hommes auraient encore besoin d'une fiction représentant la vie dans toute son intégralité.

Rousseau admet la nécessité des amusements pour l'homme, les rbonnes mœurs [tenant] plus qu'on ne pense à ce que chacun se plaise dans son état" (L.d'A., p. 298), mais rejette "tout amusement inutile [comme] un mal, pour un être dont la vie est si courte et le temps si précieux" ( $L$. d'A., p. 158). Un tel pragmatisme choque non pas en tant que solution au relativisme des goûts, mais par l'identification immédiate de la réussite de ce qui plaît à ce qui plaît en lui. Si le théâtre siège dans l'interdit, ce que présente la scène dépasse le seul effet de sa réussite. L'argument antithéâtral de la Lettre à d'Alembert sur les spectacles n'en a pas fini avec le paradoxe. Le modèle délibérant de la place publique demeure le présupposé normatif de l'argument de Rousseau. L'affaire toute entière tient à la souveraineté d'un peuple assemblé. "Le souverain ne saurait agir que quand le peuple est assemblé», écrit Rousseau dans le Contrat social (III, XII, p. 135). Au théâtre, il se produirait ce qui est interdit à la délibération d'un peuple assemblé, le public serait soumis au risque de la réflexion de soi. S'il y a un attrait naturel à vouloir s'éloigner de soi, de sa propre nature, l'imitation théâtrale a prouvé que cette tendance était déjà en dehors de ce que la culture humaine peut comporter. Autrement, c'est la déchéance. L'«indifférence" des spectacles n'est pas le fruit d'une convention particulière, mais le contre-argument qui prouve que l'homme ne peut pas se représenter lui-même sans courir le risque de voir cette représentation même échapper à sa propre présence à soi. L'immédiateté de cette présence prime sur toute proposition de plaisir. Un aperçu sur l'histoire du théâtre permettra ici de voir que l'évolution de l'enjeu de ces 
questions entre Rousseau et Schiller reflète le problème de sa fonction.

\section{b) L'histoire du théâtre par sa fonction}

Trois âges découpent l'histoire du théâtre, selon H.-G. Gadamer dans sa conférence "Au sujet du caractère de fête du théâtre». Deux traits fondamentaux parcourent la toile de fond, dessinent l'horizon d'attente de la réponse à donner à la question de sa fonction. À l'origine, le théâtre grec concentre les regards des spectateurs sur ces événements par lesquels l'existence humaine gagne sur l'éternité l'instant immobilisé de sa présence. Pour effectuer cette unification au sein même d'une mise à distance, le théâtre s'est nourri du souvenir de la fête rituelle. Élévation et communion représentent ainsi les deux éléments essentiels de son *caractère festif». De la fête, il hérite la suppression de toute intention pratique comme de toute tâche extérieure ou ultérieure. Accompli pour lui-même, l'événement théâtral, précise Gadamer, "comporte toujours quelque chose d'enlevant qui arrache ses participants à leur existence quotidienne et les élève jusqu'à une communauté qui les saisit tous" ${ }^{62}$. C'est aussi le lieu d'un auto-accomplissement qui se réalise en même temps que la représentation théâtrale unifie dans l'instant de la présence immédiate le souvenir et le présent. Cette unification est une véritable création : en elle, quelque chose de positif est présenté. Le théâtre ne s'est engagé dans la voie de l'imitation que pour proposer ses créations à la reconnaissance des spectateurs, menant à leur auto-reconnaissance. De la célébration, le théâtre conserve cette temporalité propre à un phénomène par essence récurrent. Même unique, une célébration possède la possibilité de sa répétition. Ainsi, dès sa naissance, l'instant théâtral se substitue, me semble-t-il, à la célébration dans une fête de ces événements où la vie s'assurait la permanence de l'éternité. Alors, dans l'immédiateté de leur présence les uns aux autres, les participants n'avaient pas à chercher du regard les raisons motivant leur union. Accentuant ce trait de l'immédiate communion, Rousseau propose, on le sait, comme modèle de spectacles les fêtes de lacédémone, qu'il préfère au théâtre national athénien. *C'est à Sparte, écrit-il, que dans une laborieuse oisiveté, tout était plaisir et spectacle» (L. d'A., p. 308). Ce n'est que par cette curieuse alliance du loisir et du travail qu'est possible une forme d'organisation sociale qui confère un 
caractère de fête aux activités humaines sans en excepter une seule, et qui assure en même temps qu'aucune n'échappera à la proximité des regards. En somme, toutes les critiques de Rousseau ne signifient qu'une chose: l'art de paraître a remplacé l'art d'être bien ensemble.

Le théâtre du XVIII ${ }^{e}$ siècle n'ignore pas la tension entre apparence et réalité; tout son effort est de les réconcilier. L'espace de médiation que cet art qui met en jeu le temps s'est ménagé dans la cité antique, Schiller appelle à le reconquérir dans la vie morcelée par la division du travail, du travail et du loisir. Son appel n'est pas teinté de regret, comme si la rupture n'était pas irrémédiable. Celle-ci ne peut pas être imputée à un art qui représente la quintessence des créations humaines ( $S$. , p. 821). Schiller s'appuie sur le moment d'élévation de l'art théâtral pour assurer la position transcendante de la scène. Un des traits caractéristiques du spectacle scénique est demeuré permanent, même dans les soirées les plus banales de son âge bourgeois. Selon Gadamer, le théâtre présente quelque chose qui n'est pas dû à la fantaisie d'un seul, l'auteur, ni seulement à sa production en chair et en os sous l'égide d'un metteur en scène, non, par essence, quelque chose y est évoqué qui, même inidentifié, rôde en nous tous ${ }^{63}$. Schiller cherche à assurer l'efficience de cette mise en présence, en écrivant que, si «une représentation visible a un effet plus puissant que lettre morte ou froid récit, aussi sûrement la scène a des effets plus profonds et plus durables que la morale et que les lois" ( $S$, p. 823). Aussitôt confirmée dans sa fonction séculaire, séculière, d'être présence et exclusivement présence, la scène est vouée au paradoxe de son efficience. Le théâtre moderne renverse l'antique rapport entre souvenir et présent, même si sa tâche demeure d'appliquer la leçon du temps à l'inconstance de l'instant. L'oeuvre théâtrale nouvelle comme l'œuvre déjà existante sera désormais jouée sous le registre du répertoire. Elle ne raconte plus sous le mode de la remémoration qui se suffit à elle-même, elle transforme cette remémoration en un appel à l'action dans la société. Plutôt que d'actualiser la joie de vivre d'une communauté, elle érige le bonheur de tous en promesse. Signe de ce tournant contre-nature du théâtre littéraire, ce n'est pas sans une "diabolique obstination", souligne R. Krebs, que le public, "rétif " aux intentions didactiques, exigeait "qu'on l'amuse "64. Le théâtre permanent ne s'est

63. Idem, p. 170 (tr. libre).

64. KREBS, loc. cit., p. 10. 
fixé dans la société qu'en exigeant de ses spectateurs sa transformation en instance culturelle, instance autonome de médiation entre la contemporanéité du présent et leur héritage historique et culturel. Mais la conception d'un théâtre moral est demeurée prisonnière de ces salles à l'italienne où l'espace de jeu pour les acteurs est aussi bien délimité que la place réservée aux spectateurs vraiment intéressés. Comment, en réclamant l'attention exclusive au texte, le théâtre de l'Aufklärung ne pouvait-il pas s'éloigner de la participation enjouée de tous? Mais, par là, il rapprochait son public du sentiment de soi que procure l'exercice intime de la lecture, qui lui enlevait pourtant ses participants. C'est contre cet écueil d'un auto-accomplissement dans un isolement souverain que le théâtre de notre âge, le troisième, est entré en lutte. C'est aussi grâce à un changement dans la structure de la représentation que tout son pouvoir peut être redonné au jeu.

Aujourd'hui, à en croire le manifeste du «spectateur intéressé», Bernard Dort, qui cherche à les cerner dans La Représentation émancipée, les pratiques théâtrales "récusent l'illusion d'une théâtralité qui se suffirait à ellemême " ${ }^{65}$. La vocation du théâtre naît de l' «agonistique " des différentes composantes du jeu théâtral, de leur combat pour le sens. Pour trouver sa fonction, le théâtre a besoin de "la présence "d'un spectateur" ou d'un public concret, auquel il "ne peut pas ne pas s'adresser" 66 . L' "activation du spectateur" est corrélative au changement de structure qu'a connu la représentation. Émancipée de «l'interprétation " à laquelle la soumettait la toute-puissance du metteur en scène, elle a renoncé "à une unité organique prescrite $a$ priori»; elle a reconnu la "polyphonie signifiante» du fait théâtral «ouverte sur le spectateur" ${ }^{67}$. La participation de celui-ci "s'insère, en fin de compte, dans la chaîne de construction (ou de déconstruction) du sens qui est le propre de la représentation théâtrale et que la réalité du spectacle ne saurait, à elle seule, clore ${ }^{68}$. Du spectacle pur, le jeu théâtral fait retour à l'imitation, c'est-à-dire, selon Gadamer, à cette transformation de la réalité dans laquelle la transformation fait signe vers ce qui a été transformé de cette réalité en et par elle. Cette réalité est transformée, parce que l'imitation offre au regard des possibilités inten-

65. DORT, Bernard, La Représentation émancipée, Arles, Actes Sud (Hubert Nyssen éditeur), 1988 , p. 18.

66. Idem.

67. Idem, p. 178 .

68. Idem, p. 18. 
sifiées, jamais aperçues jusqu'alors. Chaque imitation est ainsi une exploration de la réalité, une intensification des extrêmes. De retour de l'exil de la transcendance, le jeu théâtral explore de lui-même la mise en présence du contenu propre à l'événement théâtral dans l'immédiateté de la communauté des acteurs et des spectateurs. Cette mise en présence, même si elle n'appartient plus exclusivement ni au texte littéraire ni à ses créateurs, a toujours été une création. Dans la perspective de Rousseau, il aura toujours été inutile de vouloir autonomiser cette création. Dans celle de Schiller, la convocation par le jeu théâtral de toutes ses composantes ne peut résulter que d'une chose. Le théâtre ne livre combat pour le sens que parce qu'il a à faire avec notre compréhension de nous-mêmes.

\section{II. "Que pourrait être l'effet véritable d'une bonne scène permanente?"}

L'élément-clef dans l'histoire du théâtre, comme l'affirme Gadamer, se trouve donc dans cette possibilité d'offrir, pour les appeler ainsi, des propositions de sens intensifiées. Son privilège est de proposer ces possibilités qui intensifie le sens jusqu'alors aperçu de la réalité dans la communauté immédiate des acteurs et des spectateurs qui ont à faire l'épreuve de ces courageuses expériences de changement. Son efficace est de permettre ce double renvoi : l'acteur recouvre venant du spectateur ce qu'il a mis en jeu, le spectateur reçoit de la part de l'acteur des possibilités d'être pareillement risquées, mais qui l'engagent à se dépasser $^{69}$. Pour Rousseau et Schiller, cet engagement, cette participation à la représentation, n'était pas encore la clef de l'expérience du théâtre, mais elle constitue le point tournant entre un théâtre privé de public et un public réclamant du théâtre, même composé de créateurs.

Le moment de la réception, dans la conception de Jauss, apparaît comme celui qui ouvre l'expérience esthétique particulière d'une ouvre sur celle de la fonction sociale de l'art ${ }^{70}$. On ne peut de fait interdire que la transformation du monde par l'homme en son œuvre propre soit source de connaissance, mais l'exercice de tel «savoir-faire poétique" ne peut que se perdre au cours de l'histoire. On ne peut non

69. GADAMER, op. cit., p. 176

70. Cf. Jauss, « La jouissance esthétique *, loc. cit., pages 272-273 
plus empêcher qu'une quelconque forme d'art provoque une expérience perceptive, marquée par une certaine densité sensitive et une égale complexité émotive, sans autoriser la destruction de ses ouvres. Il est cependant commun aux politiques de disperser ceux qui s'assemblent, hors de toute quotidienneté, pour mettre à l'épreuve dans la communauté ainsi créée «les normes de l'action * que leur proposent les ceuvres d'art. Ainsi, des trois acteurs de la pratique théâtrale, auteurs ou comédiens, œuvres et public, Rousseau condamne ces lieux permanents qui présentent des spectacles réglés d'avance pour sauvegarder la spontanéité d'un peuple à inaugurer et à justifier politiquement ces normes. Schiller, lui, convoque ouvres et public à la défense de la scène, dont l'engagement des auteurs à sa formation esthétique garantit l'efficience permanente.

La tentative de Schiller est, selon Jauss, la première démarche en vue de restaurer la fonction communicative du théâtre, sur la base de ses effets cathartiques ${ }^{71}$. En effet, le mouvement entre production et réception passe toujours par l'intermédiaire de la communication d'une œuvre 72 . La dénonciation de Rousseau ne s'est jamais attaqué qu'à l'adhésion immédiate que provoque sa fonction mimétique au sens de Gadamer, voire à la proposition d'un moyen de "convertir" l'opinion par l'apprentissage de l'art de changer les conditions de vie.

\section{1) Considérations sur "la scène la meilleure"}

La conférence "Du théâtre considéré comme institution morale" fut prononcée à Mannheim en juin 1784 sous le titre "Que pourrait être l'effet véritable d'une bonne scène permanente?». Pour Schiller, la scène a déjà gagné son procès contre ses détracteurs et elle est "plus que toute autre institution publique dans l'État une école de sagesse pratique, un guide à travers la vie civile, une clef infaillible aux accès les plus secrets de l'âme humaine" ( $S$., p. 826). Même ses ennemis ont admis qu'elle "a un effet fondamental sur les mœurs et l'Aufklärung" et qu'elle "méritait d'avoir la préférence entre les inventions du luxe et les institutions pour le divertissement en société " (S., p. 830). S'adressant aux "esthètes " comme aux "politiques ", l'inter-

71. JAUSS, *Petite apologie de la jouissance esthérique *, op. cit. p. 134.

72. JAUSS, "Esthétique de la réception et communication littéraire "loc. cit., p. 1116. 
rogation de Schiller va du «bonheur général» à l'indépendance de la scène ${ }^{73}$.

L'œuvre théâtrale étant pour les actions humaines ce que la découverte scientifique est à la connaissance, et peut-être «le produit le plus élevé de l'esprit humain» $(S$, p. 819), Schiller pose d'abord la question de la situation effective du théâtre dans la société. Qu'est-ce donc que l'art dramatique, sinon d'être la représentation d'un «tribunal redoutable* (S., p. 823) ? Tenant le spectateur sous la protection de son instance, la scène a le pouvoir poétique de convoquer les acteurs de l'histoire et de les faire défiler devant lui. L'imaginaire théâtral puise sa force d'obligation dans sa correspondance avec «la morale de l'homme ordinaire». Schiller appuie ce rôle d'intermédiaire sur une «sensibilité » esthétique, qu'il estime aussi inépuisable qu'elle est active. Les «impressions" qu'engendre la scène, écrit-il, "sont indélébiles et au moindre effleurement tout [...] revient dans le cour de l'homme comme [si cela] reprenait vie. Aussi sûrement qu'une représentation visible a un effet plus puissant que lettre morte ou froid récit, aussi sûrement le théâtre a des effets plus profonds et plus durables que la morale et que les lois" (S., p. 823-824).

Traitant de la fonction culturelle du théâtre, la seconde partie de sa conférence engage le vrai débat avec la Lettre à d'Alembert. En effet, Schiller place la «sagesse pratique * au-dessus des codes de lois. Le rayon d'action de la scène s'élargit au-delà de celui de la justice et de la morale, parce que celle-ci accompagne ce que celles-là jugent indignes d'elles : les sentiments humains ( $S$. , p. 825). À nous, que nos vies situent entre hasard et projets, la scène donne cette préparation, cette formation de «sagesse pratique» qui ne nous laissera pas au dépourvu. Les leçons de cette célèbre "école de mœurs» nous feraient cruellement défaut dans la vie, si nous n'avions l'occasion d'exercer notre jugement, notre sens moral, devant la scène. Au théâtre, notre nature surprise nous confirme, sans que nous puissions la contredire, ce que nous ne faisions «que sentir en légers pressentiments» $(S .$, p. 826). Ce pouvoir d'éveil et de jugement apparaît clairement dans l'origine et la destination que Schiller assigne à la scène.

73. Selon Paul Menzer, les premiers écrits d'esthétique de Schiller ne sont pas nés d'un intérêt pour la théorie, mais du besoin de défendre le caractère * dissident * des personnages de ses pièces. Il tenait pour inévitable * un effet particulier et civilisateur de la scène *. Cf. Paul MENZER, "Schiller und Kant *, in Kant-Studien, Band 47, Heft 1-4, 1955-1956, I-VI, p. 113-147, VI-X, p. 234-272, ici p. 113 
À la différence de Rousseau, l'actualité du théâtre et de son caractère de médiation sont abordés par lui sans crainte pour la vie civile. Bien qu' « une irrésistible tendance pour le nouveau et l'extraordinaire, un désir de se sentir soi-même dans une condition passionnée» $(S .$, p. 821) aient engendré - ou renouvelé, comme on l'a vu - le théâtre, tous lui doivent reconnaissance pour bien autre chose.

* L'humanité et la tolérance, écrit Schiller, commencent à devenir l'esprit dominant de notre temps; leurs rayons ont pénétré jusque dans la salle des tribunaux et, encore plus, jusques au cour de nos princes. Quelle part de cet ouvrage (...) appartient à nos scènes? Celles-ci ne sont-elles pas ce qui fait connaître l'homme par l'homme et découvre les secrets rouages selon lesquels il agit?

"Ici seulement les grands de ce monde entendent ce que jamais ou rarement ils entendent : la vérité; ce que jamais ou rarement ils voient, ils le voient ici : les hommes" ( $S$., p. 828).

Dernier aperçu de Schiller, les mérites de la scène ne s'arrêtent pas à ce qui a été, mais s'étendent à ce qui pourrait être. Quand le «fervent patriote " comme le "grand penseur" auront comparé les peuples avec les peuples et les siècles avec les siècles, ne pencheront-ils pas pour inviter les poètes à prendre pour sujets les "seuls objets populaires" ( $S$. p. 829)? "Si nous pouvions connaître cela, écrit-il, avoir un théâtre national, alors nous deviendrions nous aussi une nation (id.). Entre la vie politique et la vie pratique s'établit, par l'intermédiaire du théâtre, une communication. Schiller énonce les deux phases de ce passage : par la culture morale au contact de l'histoire et par la médiation de ce qui est commun ${ }^{74}$.

«Si grand et si varié est le mérite d'une scène meilleure concernant la formation morale et un mérite non moindre lui revient concernant la pleine mise en lumière du sens commun (die ganze Aufklärung des Verstandes).

"La scène est le canal commun en lequel des meilleures parties pensantes du peuple la lumière de la sagesse se répand en faisceaux et de là, en rayons moins forts, elle se propage dans

74. Signalons qu'Habermas admire la prudence avec laquelle Schiller évite l'idéalisme de Kant et de Hegel. L'expérience de la vie moderne, entre occupations sérieuses et satisfactions sans gloire, ne requiert pas chez lui l'idée d'une *intuition * réconciliatrice, mais d'une instance esthétique de communication. Pour cela, il conjugue le concept kantien et celui de la tradition du jugement (Urteilskraft), lequel, à travers la tradition aristotélicienne, n'avait jamais tout à fait perdu sa relation avec le concept politique de sens commun (Gemeinsinns). Cf. Jürgen HABERMAS, Le Discours pbilosopbique de la modernité. Douze le fons, tr. fr. Christian Bouchindhomme et Rainer Rochlitz, Paris, Gallimard, 1988, - Digression à propos des Lettres sur l'éducation estbétique de Scbiller», p. 58 (tr. modifiee). 
tout l'État. Des concepts plus justes, des principes épurés, des sentiments plus clairs coulent d'ici à travers toutes les veines du peuple; les brumes de la barbarie et de la sombre superstition se dispersent, la nuit recule devant la triomphante lumière» $(S .$, p. 828).

\section{2) Schiller et les institutions du * sens commun *}

Schiller n'exalte l'aspect de formation du projet des "Lumières» que pour mieux contrebalancer ses effets dans le monde de l'«homme ordinaire». La jouissance d'une totalité s'offre comme le remède à la souffrance de l'homme dissocié. C'est là l'efficacité de la médiation esthétique, dont le caractère précaire est pour ainsi dire moteur. Dès ses débuts, le projet schillérien de faire concourir inévitablement l'expérience esthétique au bonheur d'un peuple passe par la reconnaissance d'un sens commun. Lui seul est le lieu où valeurs, traditions, préjugés, opinions vraies ou fausses, justes ou injustes peuvent être soumises au jugement de tous et de chacun des membres d'un peuple et non pas relever de l'exercice exclusif du savoir du législateur de ce peuple, Schiller allant même jusqu'à en faire un instrument entre les mains des "politiques" pour corriger la manie du doute du peuple "sans même en avoir l'air" $\left(S .\right.$, p. 829) ${ }^{75}$. Quoi qu'il en soit, le texte des Lettres de 1795 cautionnera l'idée de la Conférence de 1784. Dans la vingt-septième des Lettres sur l'éducation esthétique de l'bumanité, Schiller écrira encore: "Loin des arcanes de la science, le goût amène au grand jour du sens commun la connaissance et il transforme ce qui est l'apanage des écoles en un bien commun à toute société " 76 . Le théâtre connaît déjà son in térêt. Quand il isolera chacun des spectateurs qu'il rassemble devant la scène, ce sera pour faire porter en lui sa propre compétence à apprécier ce qu'elle lui propose.

\section{a) La réplique de Schiller à Rousseau}

Schiller avait lu, selon Wolgang Liepe, l'écrit de Rousseau concernant sa propre profession 77 . Déçu du verdict, le

75. Le «Législateur doit avoir recours à la ruse, à un entraînement par les sentiments, à un maniement de l'illusion et de l'image afin de faire jouer en secret l'opinion *, écrit P. Knee, loc. cit., p. 317.

76. SCHILLER, Friedrich, lettres sur l'éducation esthétique de l'bomme (17931795), tr. fr. Robert Leroux, Paris, Aubier-Montaigne bilingue, 1943, p. 355.

77. LIEPE, Wolgang, *Rousseau-Kant-Schiller *, in ses Beiträge zur Literatur - und Geistesgescbichte, Neumünster, Karl Wachholtz Verlag, 1963, p. 106-119, ici p. 116. Plutot que de faire place à la tendance au pessismisme culturel (Kulturpessimismus) héritée de Rousseau, Schiller était convaincu que la meme loi d'harmonie et de progrès anime le monde naturel et le monde humain 
jeune dramaturge ne pouvait abandonner, ni aux marges de la raison, ni aux puissances du sentiment, la part de l'homme sur laquelle le théâtre agit. Le point d'ancrage de sa défense de l'efficace de la scène, c'est l'insuffisance de toute loi, morale ou politique, à se rendre sensible dans le monde de la vie, son caractère chancelant. Cela en détermine même toute influence $(S$, p. 822). L'action véritable de la scène participe de deux mondes: la scène est un apprentissage sensible à la vie en société et elle confère la maîtrise rationnelle des passions. Sous sa lumière, les opinions et les goûts d'une communauté se rencontrent et deviennent "sens commun". "J'appelle esprit national, écrit-il, la ressemblance et la concordance des opinions et des goûts d'un peuple quant aux choses (Gegenständen), sur lesquelles une autre nation a des opinions et des sentiments différents $»(S$. , p. 830). Ce qui est commun - et qui appartient aux conventions, aux manières d'être et d'agir, avant de relever des lois, des prescriptions normatives doit circuler librement dans la communauté culturelle. Le rapport entre représentations vivantes et délibération commune se trouve soumis à la théorie esthétique.

Ainsi, l'exclusion par Rousseau d'un rapport médian entre l'art de changer l'opinion et "l'attrait du plaisir" semble ne pas être la seule réponse à la question de la fonction sociale de l'art. Schiller semble cependant ignorer le risque de voir le plaisir esthétique se dissoudre dans la subordination du jeu théâtral à l'efficace d'une scène en position de transcendance. Il n'en revendique pas moins pour la scène, qui "accomplit" quelque chose de "plus important qu'on n'est habitué à le croire" (id.), un statut purement esthétique. "La scène, argue-t-il, est l'institution où s'épousent le plaisir et l'instruction, le repos et l'effort, l'amusement et la "culture", où nulle force de [l'être] n'est tendue au détriment des autres, où nul plaisir n'est goûté aux dépens du tout» $(S$. , p. 831). Mais s'agit-il en réalité de la possibilité de se servir du pouvoir du théâtre ou de celle de le faire servir? L'expérience esthétique où s'éprouve la jouissance de soi-même dans la jouissance de l'autre, et que Jauss appelle une découverte de l'altérité, demeure-t-elle, dans l'une comme dans l'autre possibilité, irréductible?

\section{b) Le différend de Rousseau et Schiller sur l'institution théâtrale}

La métaphore du redoutable tribunal, qui compense les défauts "de la balance et de l'épée» et substitue à la séduc- 
tion «qui appelle de loin" de la religion la «concrétude» et la "présence vivante * de l'histoire m'apparaît être en superposition dans le texte de Schiller. Elle dissimule et montre à la fois que coïncident l'origine et la destination de la scène. Schiller fait valoir le pôle opposé d'une instance esthétique de communication : non plus le rapport social garanti par l'universalité du droit et vécu dans la solidarité affective, mais l'individualité élevée, en principe, au-dessus des dualismes entre nature et culture, sentiment et raison ${ }^{78}$. L'autonomie de la scène correspond, droit pour droit, à l'autonomie de la personne individuelle, dont Rousseau demeure l'instigateur ${ }^{79}$. La société, lieu des antagonismes, ne représente plus la source de réalisation du bonheur humain, mais la scène s'offre d'elle-même à la remplacer à ce titre. En effet, elle est ce lieu où "passent devant l'homme dans des milliers d'images compréhensibles et vraies", vice et vertu, félicité et misère, folie - ou bêtise - et sagesse, écrit Schiller ( $S$, p. 822). Ce qui vient résoudre la dichotomie pratiquée par Rousseau entre nature et culture, ce sont à la fois la constitution humaine, avec sa nature sensible et intelligible, et l'instinct d'activité, avec sa tendance à se jeter "sans frein" dans les distractions comme dans les occupations $(S$. , p. 830). La conciliation de la raison et du sentiment s'avère esthétiquement possible, puisque le «sens esthétique» prouve la continuité existant entre l'origine naturelle de la scène et sa destination rationnelle :

* Épuisé par les grands efforts de l'esprit, las des monotones et souvent accablantes affaires de son travail, enfin rassasié dans sa sensualité, l'homme devait ressentir un vide dans son être, vide qui était contraire à son éternel instinct d'activité. Notre nature, incapable de persister plus longtemps dans l'état de l'animal comme de poursuivre les travaux plus fins de l'intelligence, exigeait une condition intermédiaire qui réunisse ces deux tendances extrêmes et contraires, qui disposât à une harmonie plus douce cette forte tension et qui ménageât un passage réciproque d'une disposition à l'autre. Ces résultats, en fait, c'est le sens esthétique ou encore le sentiment pour le beau qui les provoque» ( $S$., p. 821).

Si, avec la Lettre à d'Alembert, l'art théâtral avait dû essuyer sa "plus dure attaque» $(S$., p. 820), l'esthéticien allemand a bien mesuré sa riposte. Le premier souci d'un «sage législateur» devrait être de choisir entre deux effets le

78. Schiller travaillait, selon Liepe, à réaliser «l'unité de la nature et de la culture, du sentiment et de l'intelligence, dans la personne individuelle de l'homme *, op. cit., p. 114.

79. Cf. Cassirer, op. cit., p. 106-107: le bonheur supreme est celui que confère la libre * personnalité *. 
plus durable ( $S$. p. p. 821). À la justice et à la religion, il préférera l'action « véritable * du théâtre. C'est dans le miroir que dresse la scène devant ceux qu'elle assemble que le législateur «découvre combien servilement la grande majorité du peuple demeure prisonnière des chaînes du préjugé et de l'opinion" ( $S$., p. 828). Quand Rousseau compte sur l'équilibre des avantages et des inconvénients : quand «le bien surpasse le mal, la chose [devant] être admise malgré ses inconvénients » (L.d'A., p. 275), ce calcul n'est pas selon Schiller le plus pratique. Non content d'avoir désarmé les penchants de leurs peuples, la "singulière catégorie d'hommes" qui les gouvernent se servira de ces penchants comme des instruments d'un plan plus grand et s'appliquera à les transformer en sources de bonheur $(S$, , p. 821). Pour corriger l'opinion qui gouverne moralité et socialité, Schiller préfère, à l'art bien gris de changer l'opinion publique, la nouveauté, l'inédit de la rencontre chaque fois différente entre une scène et un public qu'est chaque représentation théâtrale. Loin de contribuer à la dégradation de la condition humaine, l'action de la scène la restaure plutôt : elle conserve ce qu'il y a de meilleur en l'humanité tout en proposant de juger ce qu'elle comporte de pire. Elle offre à la représentation publique ce qu'une communauté a la sagesse de taire dans la vie, mais tient à entendre dire en ce lieu qu'elle appelle depuis ses origines.

Le législateur, le gouvernant et le peuple se retrouvent devant la scène et composent ce public, que dans la perspective schillérienne personne ne peut craindre ni ignorer. Hommes et femmes "de tous les milieux, de toutes les zones, de tous les états" viennent s'y affranchir "de toute chaîne " et fraterniser "en s'oubliant eux-mêmes et le monde» ( $S$. p. 831). Cette solution n'est pas étrangère à la dernière proposition de Rousseau. À sa décharge, quelle notion du sentiment ouvre, en effet, sans contrainte et sans souci d'utilité, un «canal commun"? La scène est-elle bien ce lieu où peut être élevée à son plus haut degré la concordance des opinions et des goûts ? Si la scène "traverse tout le domaine du savoir humain, épuise toutes les situations de la vie (..), porte la lumière dans tous les replis du cœur $(\ldots)$, réunit en elle tous les états et toutes les classes " $(S$. p. 830), peut-elle à elle seule ouvrir cette voie vers le «bonheur général» que "le philosophe et le législateur d'une institution publique $n^{\prime}$ 'ont qu'à suivre? La position transcendante, qui l'autonomise, ne peut suffire à empêcher que ce ne soit qu'en se faisant public que le peuple participe au spectacle de lui-même. Le rêve le plus difficile de Schiller a 
les accents d'une solidarité perdue: "Chacun jouit en luimême du ravissement de tous, qui le fortifie et l'embellit sous cent regards qui tombent sur lui, et sa poitrine ne donne place maintenant qu'à un unique sentiment - et c'est celui-ci : d'être un homme» $(S$., p. 831).

\section{c) L'ultime proposition de Rousseau}

Aux limites extrêmes de leurs projets, la «vérité de pratique importante à tout un peuple" de Rousseau ( $L$. d'A., p. 143) rejoint la "sagesse pratique" de Schiller. Un peuple pourrait s'approprier, au double sens du mot, cette sagesse, cette vérité, pour tracer la frontière entre ses plaisirs et ses devoirs, entre esthétique et vie civile. La question alors est celle de savoir si, comme l'affirme Schiller, les médiations proviennent du même lieu qui les rend vivantes. En somme, pourquoi la tâche la meilleure d'un législateur à l'égard d'un peuple demeure-t-elle, selon Rousseau, de lui accorder des spectacles? En quelque sorte, Rousseau appuie sa proposition sur le souvenir qu'il a gardé d'une fête improvisée sur la place Saint-Gervais à Genève ( $L$. d'A., p. 310, note), comme sur une «norme" intérieure d'après laquelle, selon Starobinski, il «juge et condamne les autres spectacles ${ }^{80}$. Et «il en faut beaucoup», écrit-il :

«C'est dans les Républiques qu'ils sont nés, c'est dans leur sein qu'on les voit briller avec un véritable air de fête. À quels peuples convient-il mieux de s'assembler souvent et de former entre eux les doux liens du plaisir et de la joie, qu'à ceux qui ont tant de raisons de s'aimer et de rester à jamais unis" ( $L$. d'A., p. 297)?

Il en va ici comme de l'envers esthétique de la fusion des volontés particulières dans la volonté générale, qui force à être libre (CS, I, VII, p. 64). En concurrence avec le mode de médiation du théâtre où chacun a le regard fixé sur ce que tous regardent, Rousseau redonne au spectacle le caractère de la fête. Ce sont des fêtes que méritent les peuples heureux : «C'est en plein air, c'est sous le ciel qu'il faut vous rassembler et vous livrer au doux sentiment de votre bonheur» (L. d'A., p. 297-298). La fête est l'exact divertissement qu'un peuple peut approprier à son activité, entre ses travaux et ses plaisirs. Elle seule est collective, supprimant les barrières et les distinctions, parce qu'elle est à elle-même son propre spectacle (L. d'A., p. 310). L'impératif de la transparence oblige enfin le peuple à se voir dans la réalité 
du bien qu'il a accompli; ce n'est donc pas lui-même qui se trouve représenté à la fête. L'élément de communion, Gadamer le dit avec raison, ne suffit pas seul à la mise en présence d'une création propre au caractère festif du théâtre, à sa communication. Il manquera toujours cette distance à l'égard de soi que Rousseau attribue ici aux organisateurs. En effet, il demande: "quels seront enfin les objets de ces spectacles. Qu'y montrera-t-on? Rien, si l'on veut" (L.d'A., p. 298). La fête se propose comme son propre contenu certes, mais c'est parce qu'elle s'inscrit dans la régularité d'un ordre d'alternances entre travail et divertissement. S'adressant aux organisateurs, faites "encore mieux, dicte Rousseau : donnez les spectateurs en spectacle; rendez-les acteurs eux-mêmes; faites que chacun se voie et s'aime dans les autres, afin que tous soient mieux unis» $(i d$.$) . À la fête$ doit triompher le sentiment, mais Rousseau est demeuré irrésolu sur son statut esthétique. La solution au problème - politique - du bonheur passe-t-elle enfin par l'esthétique, comme le voudrait Schiller?

\section{3) La conquête de l'enjeu esthétique}

La proposition de Rousseau de donner la préséance au sentiment sur la raison a commandé une élucidation esthétique du monde de la vie, mais il n'a pas voulu fonder cette préséance sur la compréhension de soi d'une communauté ayant joui des mêmes choses. Un peuple, privé de médiations, on l'a vu, ne peut de lui-même s'approprier la com. pétence de distinguer entre sentiment et norme. Le projet de Schiller exige en retour de reconnaître les composantes effectives de l'expérience esthétique. La jouissance esthétique, celle qui passe par l'imaginaire, serait même la «seule», selon H.R. Jauss, ayant "permis, au long d'une expérience séculaire, de dégager la fonction émancipatrice de l'art"81.

Ainsi, en se tenant dans l'espace théâtral que la Lettre et la Conférence ont ouvert, il est possible d'évaluer à nouveau l'enjeu d'une fonction esthétique enracinée dans l'expérience de jouissance esthétique, telle que la conçoit Jauss. La Conférence de Schiller a moins à faire avec la lettre du "pessimisme culturel" qu'avec l'esprit de l'ultime proposition de Rousseau. La jouissance du travail tendant à se dissocier de la jouissance de ses fruits, comme Schiller l'affirme le premier ${ }^{82}$, la vie civile elle-même en vient à

81. JAUSS, «Petite apologie de l'expérience esthétique , loc. cit., p. 128

82. Cf. Jauss, « La jouissance esthétique , loc. cit., p. 265 et la sixième des Lettres, op. cit., p. 105-113 surtout. 
s'opposer à la jouissance esthétique. La voie la plus accessible et la plus secrète "vers l'intelligence et le cour" ( $S$, p. 830) n'est praticable, selon Schiller, que pour autant que la scène mette en correspondance l'individualité esthétique et un groupe culturel, fasse agir de concert le plaisir esthétique et la fonction sociale de l'art. Mais Rousseau ne prescrit pas à ceux qui ont le sentiment d'être dans l'métat d'homme " l'art pour leur assurer la sorte de bonheur qui leur convient. Sa Lettre a plutôt révélé la présence de "contradictions dans le procès de communication entre le théâtre et la société " 83 . Engoncée dans la mimèsis, la représentation théâtrale prouverait son inefficacité politico-morale $^{84}$. Solution non moins paradoxale, la praxis théâtrale schillérienne ne semblerait avoir d'efficacité qu'en opposant la vie civile à elle-même, alors que le «sentiment d'être un homme» devait se concilier avec elle sous les feux de la rampe.

\section{a) Considérations sur l'actualité du plaisir}

La définition de Jauss de «l'attitude de jouissance esthétique», qui libère de quelque chose et pour quelque chose $^{85}$, s'ancre dans la tradition officieuse de l'expérience esthétique. Opposée à la nécessité pratique du travail et aux besoins naturels de la vie quotidienne dont elle a pour fonction reconnue de distraire, la jouissance esthétique n'a pas, selon lui, à être, en tant que divertissement, exclue des domaines du connaître et de l'agir. La notion d'efficacité en esthétique est relayée, chez lui, par celle d'«intérêt esthétique», c'est-à-dire cette orientation vers l' «effet esthétique» qui fait appel à une libre prise de position, mais qui demeure en même temps commandée par "l'objet" que le plaisir "découvre de plus en plus" à celui qui jouit de lui-même en éprouvant cette découverte ${ }^{86}$. C'est autour de la jouissance esthétique que s'articulent les trois actes caractéristiques de l'expérience de l'art. Le jugement esthétique souverain n'est en somme que le point culminant d'une modernité qui a pensé l'art sous le signe de la finalité, plutôt que de faire justice à l'ouverture concrétisante qui en est l'aspect communicatif.

83. FONTIUS, Martin, *Einleitung* zur Theater und Aufklärung, Dokumentation zur Ästhetik des französischen Theaters im 18. Jahrhundert, herausgegeben und komentiert von Renate Petermann und Peter-Volker Springborn, München und Wien, Carl Hanser Verlag, 1979, p. 7-39, ici p. 33.

84. Idem

85. IAUSS, « La jouissance esthétique *, loc. cit., p. 273

86. Idem, p. 270. 
En effet, selon Habermas, la théorie de la réception représente la suggestion d'«un moyen d'échapper aux apories de la modernité culturelle ${ }^{87}$. L'autonomisation de la scène n'est qu'un des corollaires de l'autonomisation des sphères de la science, de la morale et de l'art, laquelle préside à la triple exclusion de l'expérience de l'art du connaître, de l'agir et du monde de la vie. La réconciliation de ces trois modes de l'expérience humaine a, pour lui, à faire avec le renouvellement de l'orientation de l'expérience esthétique. Celle-ci, n'étant plus centrée sur la formulation critique d'un jugement de goût, peut être "prise en charge par une histoire individuelle ou incorporée dans une forme de vie collective " 88 . La réception des ceuvres d'art par le "profane" s'accompagne alors d'un changement dans l'interprétation de celles-ci, l'expérience esthétique acquérant une «fonction d'exploration capable d'orienter l'existence ${ }^{89}$. Aux yeux du théoricien de la société, l'expérience esthétique se révèle de ce fait porteuse d'une fonction d'intégration qui se manifeste dans la façon dont les interprétations des besoins à la lumière desquels le monde est perçu, les démarches cognitives et les attentes normatives «renvoient» les unes aux autres ${ }^{90}$. Dernier mot de la théorie de l'art de Habermas : la fiction fait montre d'une "fonction d'ouverture au monde"91. C'est ainsi, à mes yeux, dans le passage de l'exercice vivant d'une compétence esthétique d'exploration des possibles insoupçonnés que proposent les œuvres théâtrales à sa mise en forme théorique, que le caractère irréductible de la jouissance esthétique risque le plus. Même la position de Jauss, en reposant la question de la liberté de l'expérience esthétique, dissipe à grand-peine l'ombre que jette sur cette liberté le différend entre Rousseau et Schiller.

\section{b) L'inclination sensible de la raison}

Devant le fond de scène des possibilités de la jouissance esthétique, les considérations de Schiller et de Rousseau sur l'efficace scénique rendent perceptible combien il est facile de transgresser les limites de l'affinité des goûts et des mours comme celles de l'orientation de l'exploration des

87. HABERMAS, Jürgen, *La modernité un projet inachevé*, in Critique, 413, octobre 1981 , p. $950-967$, ici p. 963

88. Idem, p. 964 .

89. Idem

90. Idem, p. 965 .

91. ROCHLITZ, Rainer, - Des philosophes allemands face à la pensée française *, in Critique, 464-465, janvier-février 1986, p. 33 
différences. Pour éviter ce risque, Rousseau a-t-il le dernier mot avec la formulation d'un critère, "gris", du plaisir approprié ? Schiller cherchait, selon Liepe, à conjuguer l'individualité, l'universalité et la totalité dans l'intensité92. En effet, il écrit :

"Quand le monde et les affaires nous dégoûtent, quand mille charges pèsent sur (nous) et que les travaux de notre profession menacent d'étouffer notre sensibilité, c'est alors que nous reçoit la scène - et dans ce monde factice nos rêves mettent en suspens le monde réel, nous sommes rendus à nous-mêmes, notre sensibilité s'éveille» (S. , p. 831)

Toutefois, le "canal commun" que Schiller cherche à ménager pour rétablir la communication entre les sphères d'un monde souffrant des dichotomies de l'expérience humaine m'apparaît relever d'un concept de communication moins dialogique qu'il ne le suppose. La "scène la meilleure " se montre moins "programmatique " que ne le prédit Habermas pour les Lettres sur l'éducation esthétique 93. Celle-ci n'est pas encore totalement engagée dans l'effectuation de la raison, même si elle plaide déjà en faveur de la "résurrection du sens communautaire détruit ${ }^{94}$. La conférence de l'esthète Schiller n'est pas encore l'œuvre du politique Schiller qui a séjourné dans les chemins des dualismes kantiens. Certes, l'autonomie de la scène se gagne par l'opposition constante de sa fiction au monde réel, mais chacun risque de demeurer prisonnier de sa propre conscience de l'apparence en tant qu'apparence. Je préfère voir dans ce bref texte de Schiller ce qui se révèle encore novateur. Schiller considérait le plaisir esthétique comme la conséquence du rapport sensible de la fiction avec la raison. Pour lui, la communication du texte de l'imaginaire humain ne nécessite pas d'être basée sur la connaissance rationnelle des effets du jeu théâtral ; la scène la réalise depuis toujou rs. Sans cela, la jouissance esthétique de la communauté des spectateurs s'éloigne d'autant de ceux qui en sont exclus, parce qu'ils ont le sentiment d'ignorer à quelle assemblée ils ont affaire.

92. LIEPE, op. cit., p. 109 (tr. libre)

93. HABERMAS, Jürgen, Le Discours pbilosopbique de la modernité. Douze leçons, p. 54 .

94. Idem, p. 57 


\section{La mise en scène de la jouissance esthétique}

«Mon goût pour le théâtre a toujours été partagé entre deux sentiments : le désir d'une communion et la jouissance d'une différence", écrit Bernard Dort dans La Représentation émancipée ${ }^{95}$. La réponse à la question de la fonction sociale de l'art relève-t-elle ultimement du public ou de ce que Schiller nomme ironiquement «le moins corruptible des juges, la postérité " ( $S$., p. 820) ? Dernier argument puisé à l'expérience de la représentation théâtrale : l' 'interdit » du théâtre témoigne-t-il de la présence dans les ouvres théâtrales elles-mêmes de la compétence pour un public, une société, d'évaluer quels divertissements, qui portent avec eux leur distanciation d'avec lui, peuvent lui convenir à elle ? Autrement dit, comment ces événements esthétiques qui approfondissent la continuité de chacun avec lui-même et le monde dans lequel il vit s'approprient-ils leur public et s'approprient-ils la société ? Ces deux mouvements, qui font l'efficace de toute proposition intensifiée d'exploration, le chapitre de Vérité et métbode de Gadamer, portant sur "l'exemple du tragique " 96 , permet de les mettre en parallèle avec les deux traits fondamentaux liés à l'histoire de la fonction du théâtre. Si les membres d'une société, ni plus ni moins morale que celle de Genève au XVIII ${ }^{e}$ siècle, communient dans le jeu théâtral, c'est que quelque chose d'autre entre en jeu que le simple «espace théâtral, ce lieu à la fois autonome et affranchi des lois du monde par sa propre normativité. Le paradoxe essentiel de la mise en présence de la présence pour la présence, n'est-ce pas l'expérience d'une certaine forme de subsistance? Ce qui se déroule sur la scène, c'est, écrit Gadamer, "un cercle clos de significations qui se refuse à toute intrusion et à toute intervention extérieure "97. Ce qui est expérimenté comme tragique, même dans la vie, doit simplement «être accepté". La distance que la scène se gagne dans le monde de la vie est seulement relative à l'inclusion de ses spectateurs consentants. Le théâtre, en tant qu'art d'interprétation, Schiller l'a bien senti, est le modèle premier de toute forme de re-présentation, en ce qu'il engage le représentant et le représenté.

95. DORT, Bernard, op. cit., p. 11

96. GADAMER, Hans-Georg, Vérité et méthode, tr. fr. partielle d'Étienne Sacre, Paris, Seuil, 1976, p. 61.

97. Idem, p. 57 (tr. modifiee, J.C. PETIT, in Plsilosopbiques, X, 1, avril 1983 p. $153-176)$ 
"Ce n'est jamais uniquement", écrit Gadamer, "dans un monde étranger de magie, d'ivresse, de rêve que l'exécutant, le créateur d'images ou le spectateur sont ravis ; c'est toujours à leur monde propre qu'ils sont incorporés de façon authentique, dans la mesure où ils se reconnaissent plus profondément en lui. Il subsiste une continuité de sens qui relie l'œuvre d'art au monde de l'existence, et dont même la conscience aliénée d'une société cultivée ne se détache jamais 98 .

Mais si le jeu théâtral est facteur de communion par l'ouverture de sa structure représentative, son efficace n'apparaît-il pas ici fonction de sa fermeture sur le plaisir qu'il promet? Ce n'est jamais que dans un jeu d'ombres et de lumière qu'il propose l'ouverture de soi à l'autre. Le dialogue entre Schiller et Rousseau rend manifeste l'interdit auquel se livre la scène elle-même. Du moins, est-ce la leçon que nous pouvons tirer de la position de Habermas quand, selon lui, "l'emploi esthétique (du langage) suppose la suspension ou la neutralisation de la force illocutoire qui contraint les sujets à réagir par des décisions ou des prises de position ${ }^{99}$. Cela semble condamner le jeu théâtral à l'inefficacité. Comme l'écrit Rousseau, chacun n'a qu'à «consulter l'état de son coeur * au sortir du spectacle ( $L$. $d$ 'A. p. 164). Mais cette inefficacité n'atteste-t-elle pas une potentialité différente? Le cadre théorique de la communication esthétique ne peut jamais représenter pour l'expression des désirs et des besoins la médiation "la meilleure", car, en somme, tous cherchent aussi ailleurs à se garantir de la cécité de leurs désirs comme à garantir la liberté de leurs besoins. Le problème de conciliation de Rousseau et de Schiller se retrouve dans celui de Habermas, Jauss et Gadamer. Si Habermas cherche avec Schiller à coordonner les fonctions d'exploration et d'orientation de l'art au bénéfice d'une fonction d'intégration, Rousseau plaide en faveur d'une certaine réciprocité qui vaudrait dans chaque cas individuel entre l'irréductibilité de la jouissance esthétique selon Jauss et l'appartenance de l'expérience esthétique à une communauté de signification chez Gadamer.

Le cas d'application qu'a représenté Genève témoigne des difficultés de formulation du critère de l'«approprié». Les hésitations de Rousseau à étendre ce critère au domaine esthétique ont montré qu'il fallait en "convertir" le sens. C'est ainsi qu'appropriation et partage sont apparus comme les deux mouvements concomitants de la jouissance chez 
Jauss. L'élucidation de l'action de «la scène la meilleure», acquise avec Schiller, fournit alors l'élément de réciprocité entre ce qui est partageable en commun et ce qui n'est applicable qu'à soi seul, pour soi seul. La représentation que propose à la société la scène n'est acceptable que si l'ouverture qu'elle pratique dans l'espace social et dans le temps humain laisse son public libre de s'y engager. Lui-même libre d'adopter une position transcendante, le jeu théâtral ouvre sur une expérience où le spectateur est, pour Gadamer, depuis toujours déjà engagé. La distance du sentiment, c'est toujours l'espace que la raison peut franchir pour y gagner le temps de sa subsistance. Enfin, qu'il en soit dans la vie comme dans la fiction, c'est ce qu'exige la jouissance esthétique, parce que la fiction se situe aussi à l'horizon du monde de la vie.

Faculté de philosophie, Université Laval, Québec. 